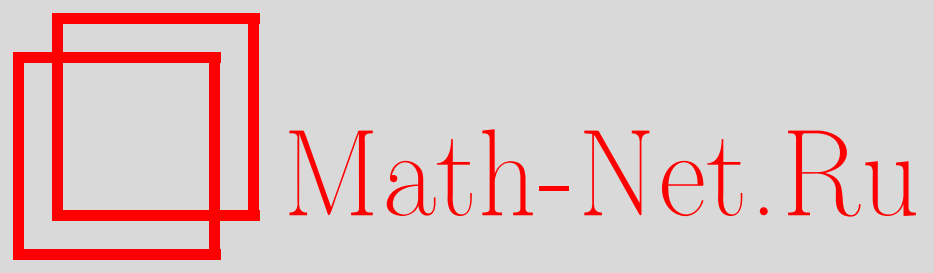

В. В. Яновский, А. В. Тур, Ю. Н. Масловский, Заряженная композитная частица в постоянном электрическом поле, ТМФ, 2013, том 175, номер 2, 247-278

DOI: https://doi.org/10.4213/tmf8418

Использование Общероссийского математического портала Math-Net.Ru подразумевает, что вы прочитали и согласны с пользовательским соглашением http://www . mathnet.ru/rus/agreement

Параметры загрузки:

IP: 35.174 .16 .151

26 апреля 2023 г., $17: 26: 59$

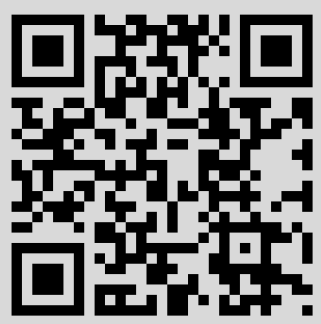




\section{ЗАРЯЖЕННАЯ КОМПОЗИТНАЯ ЧАСТИЦА В ПОСТОЯННОМ ЭЛЕКТРИЧЕСКОМ ПОЛЕ}

Рассмотрено движение заряженной композитной частицы в постоянном электрическом поле. С использованием биллиардного формализма установлены точные законы движения такой частицы с небольшим числом внутренних степеней свободы. Предложен обобщенный прием Шварца спрямления траекторий при наличии поля. В рамках биллиардного формализма получены режимы движения композитной частицы с двумя внутренними степенями свободы в постоянном поле.

Ключевые слова: частица с внутренними степенями свободы, постоянное поле, биллиард.

DOI: $10.4213 / \operatorname{tmf} 8418$

\section{1. ВВЕДЕНИЕ}

В настоящее время интенсивно развивается изучение свойств разнообразных наносистем (см., например, монографию [1]). Такие системы обладают малыми, но конечными размерами и, как правило, небольшим числом внутренних степеней свободы. Это делает актуальным изучение свойств частиц, которые зависят от их внутреннего устройства и размеров. Такие частицы уже нельзя рассматривать как точечные, и их устройство, т. е. внутренние степени свободы, может приводить к необычному поведению. Особый интерес представляют частицы с небольшим числом внутренних степеней свободы, у которых свойства зависят от числа внутренних степеней свободы. Простая модель структурно-сложной частицы была предложена в работе [2]. В этой работе также были изучены необычные свойства отражения от барьеров. Интересно отметить, что модель такой композитной частицы реализуется экспериментально в виде нанотрубки с фулереном внутри [3]-[5].

Другой интересный нанообъект, к которому применим используемый в настоящей работе подход, - это искусственные молекулярные соединения, такие как ротаксаны [6]. Название этих образований происходит от латинских слов rota - колесо и axis,

${ }^{*}$ Институт монокристаллов НАН Украины, Харьков, Украина.

E-mail: yanovsky@isc.kharkov.ua

${ }^{\dagger}$ Université de Toulouse [UPS], CNRS, Institut de Recherche en Astrophysique et Planétologie, Toulouse, France 
что означает ось. Ротаксаны состоят из гантелеобразной молекулы с большими группами на концах, которые предотвращают соскальзывание надетой на нее циклической молекулы [6]. Гантелеподобная компонента ротаксанов и циклическая молекула связаны механически без участия химической связи. Циклическая молекула может перемещаться вдоль оси и вращаться. Концевые препятствия не позволяют "колесу" покинуть ось, и поэтому такую связь можно рассматривать как топологическую. Впервые такие соединения были синтезированы в 1967 г. [7]. Один из важных успехов в использовании ротаксанов состоит в достижении рекордных значений плотности записи информации [8], [9]. Кроме этого, ротаксаны играют важную роль при конструировании молекулярных машин. Для этого имеется несколько важных причин. Прежде всего, наличие механических или топологических связей создает возможности для разнообразных расположений и соединений молекулярных деталей. При этом топологическая связь гарантирует устойчивость конструкции. Это делает возможным управление компонентами с помощью внешних воздействий. Поэтому интересно изучить влияние внешних воздействий на динамические режимы композитных частиц. Примером одного из простых воздействий, которое легко реализовать, может служить постоянное электрическое поле, разумеется, в случае наличия заряда у компонент композитной частицы. Изучение влияния этого фактора на заряженную композитную частицу и проводится в настоящей работе.

Мы предлагаем обобщение биллиардного формализма для описания движения заряженных композитных частиц в электрических полях. Основное внимание уделено частице с одной внутренней степенью свободы в постоянном электрическом поле. Получены все возможные режимы движения и установлены критерии их реализуемости. Общие принципы этого формализма переносятся и на более сложные случаи. Рассмотрен случай композитной частицы с двумя внутренними степенями свободы и исследованы возможные режимы движения. Обнаружен солитоноподобный режим движения. В общем случае для такой композитной частицы становится типичным хаотический режим движения. Показано, что биллиардный формализм позволяет установить основные особенности движения такой частицы и приводит к ряду интересных проблем. Используя предложенный в работе обобщенный прием спрямления траекторий, мы показываем, что и в этом случае движение заряженной композитной частицы сводится к движению точечной частицы в безграничной среде, но в поле потенциальной энергии определенного вида.

\section{2. БИЛЛИАРДНЫЙ ФОРМАЛИЗМ}

Рассмотрим движение композитной частицы с одной внутренней степенью свободы в постоянном электрическом поле $E$. Пусть оболочка частицы представляет собой цилиндр длины $L$ с закрытыми торцами, имеющий массу $m_{1}$ (см. рис. 1$)$. Внутри оболочки свободно движется внутренняя частица массы $m_{2}$. Предположим сначала, что заряд $e_{2}$ имеет только внутренняя частица, а оболочка нейтральна или незаряжена $\left(e_{1}=0\right)$. Для описания общих закономерностей движения такой частицы используем биллиардный подход [2]. Начнем с уравнений движения для внутренней частицы и оболочки:

$$
m_{1} \ddot{x}_{1}=0, \quad m_{2} \ddot{x}_{2}=E e_{2},
$$




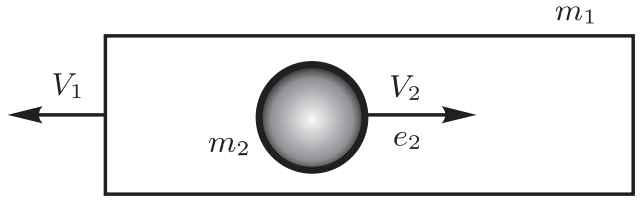

Рис. 1. Схематическое устройство композитной частицы, движущейся в постоянном электрическом поле $E$. Внутренняя частица с зарядом $e_{2}$ имеет массу $m_{2}$. Оболочка нейтральна, $e_{1}=0$, и имеет массу $m_{1}$. Скорость оболочки обозначена через $V_{1}$, а скорость внутренней частицы - через $V_{2}$.

где $x_{1}$ - координата положения центра оболочки, а $x_{2}$ - координата внутренней точечной частицы. Далее перейдем к новым координатам $\tilde{x}_{1}=\sqrt{m_{1}} x_{1}$ и $\tilde{x}_{2}=\sqrt{m_{2}} x_{2}$, которые далее будем называть биллиардными. Уравнения движения в биллиардных координатах принимают вид

$$
\sqrt{m_{1}} \ddot{\tilde{x}}_{1}=0, \quad \sqrt{m_{2}} \ddot{\tilde{x}}_{2}=e_{2} E .
$$

Рассмотрим конфигурационное пространство композитной частицы. Ясно, что все возможные положения такой системы определяются неравенством

$$
\left|\frac{\tilde{x}_{1}}{\sqrt{m_{1}}}-\frac{\tilde{x}_{2}}{\sqrt{m_{2}}}\right| \leqslant \frac{L}{2},
$$

которое означает, что внутренняя частица не может выйти за пределы оболочки. Легко понять, что конфигурационное пространство состояний такой системы есть полоса ширины $L_{*}=L \sqrt{m_{2}}$, показанная на рис. 2. Эта полоса наклонена по отношению к оси $\tilde{x}_{1}$ под углом $\alpha$, который определяется отношением масс оболочки и внутренней частицы: $\operatorname{tg} \alpha=\sqrt{m_{2} / m_{1}}$.

Таким образом, движение оболочки и частицы согласно уравнениям (2) определяет параметрическую кривую $\left(\tilde{x}_{1}(t), \tilde{x}_{2}(t)\right)$ в конфигурационном пространстве до момента достижения частицей границы этого пространства (см. рис. 2). После этого в соответствии с законами столкновения масс оболочки и внутренней частицы происходит изменение их скоростей. Используя скорости как начальные условия при решении уравнений (2), снова получим параметрически заданную кривую (см. рис. 2).

В биллиардных координатах отражение траектории от стенок эффективного бильярда в полосе или от границ конфигурационного пространства будет абсолютно упругим: угол отражения траектории от границы биллиарда равен углу падения. Физически это легко объяснить. Дело в том, что законы столкновения масс являются локальными и не зависят от наличия или отсутствия поля. Поэтому доказательство в биллиардных координатах равенства угла падения углу отражения повторяет доказательство в случае отсутствия электрического поля [2]. Это означает, что движение композитной частицы с одной внутренней степенью свободы в постоянном электрическом поле эквивалентно движению заряженной точечной частицы в биллиарде в постоянном электрическом поле, направленном вдоль оси $\tilde{x}_{2}$. 


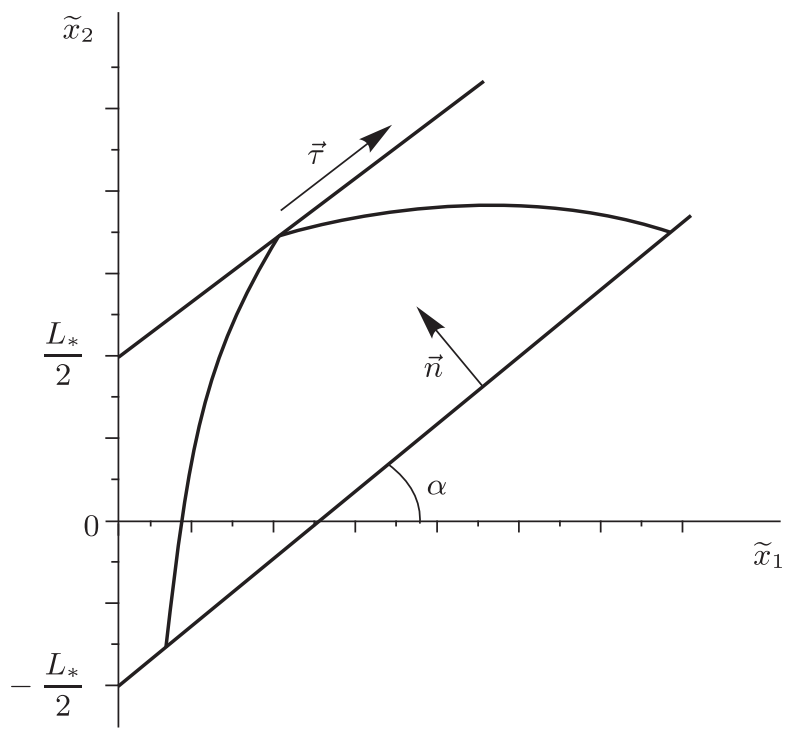

Рис. 2. Конфигурационное пространство композитной частицы. Достижение траекторией нижней границы означает столкновение внутренней частицы с правой стенкой оболочки, достижение верхней границы - столкновение с левой стенкой оболочки.

Такое специальное направление электрического поля связано с отличием от нуля заряда внутренней частицы. Далее данную точечную частицу мы будем называть эффективной частицей.

Начнем с выяснения всех возможных режимов движения такой композитной частицы. Более общий случай с отличным от нуля зарядом оболочки также обсудим ниже.

\section{3. РЕЖИМЫ ДВИЖЕНИЯ КОМПОЗИТНОЙ ЧАСТИЦЫ С ОДНОЙ ВНУТРЕННЕЙ СТЕПЕНЬЮ СВОБОДЫ}

Рассмотрим движение композитной частицы в рамках биллиардной идеологии. Основное внимание в данном разделе сосредоточим на эволюции скоростей оболочки и внутренней частицы. Описание скоростей удобно осуществить, переходя к дискретному времени. Дискретизация достигается определением или фиксацией скоростей сразу после столкновения траектории с границами биллиарда. Между столкновениями изменение скоростей универсально: скорость оболочки сохраняется, а скорость внутренней заряженной частицы меняется равноускоренно. В таком дискретном времени законы изменения скоростей можно описать с помощью отображений.

Пусть на некотором шаге эволюции биллиардная траектория отражается в точке $\left(\tilde{x}_{10}, \tilde{x}_{20}\right)$ от "нижней" границы биллиарда (см. рис. 2). Это означает, что начальные 
координаты точки отражения удовлетворяют условию

$$
\frac{\tilde{x}_{20}}{\sqrt{m_{2}}}=\frac{\tilde{x}_{10}}{\sqrt{m_{1}}}-\frac{L}{2}
$$

Обозначим скорость внутренней частицы сразу после отражения через $\widetilde{V}_{2, n}$, а скорость оболочки - через $\widetilde{V}_{1, n}$. Далее траектория эффективной частицы достигает верхней границы биллиарда. Траекторию на этом этапе можно найти, используя точные решения уравнений (2). Действительно, интегрируя тривиальные уравнения (2), получим для скоростей зависимости

$$
\widetilde{V}_{2}(t)=\frac{e_{2} E}{\sqrt{m_{2}}} t+\widetilde{V}_{2, n}, \quad \widetilde{V}_{1}(t)=\widetilde{V}_{1, n},
$$

а для изменения координат со временем - зависимости

$$
\tilde{x}_{2}(t)=\frac{e_{2} E}{2 \sqrt{m_{2}}} t^{2}+\tilde{V}_{2, n} t+\tilde{x}_{20}, \quad \tilde{x}_{1}(t)=\tilde{V}_{1, n} t+\tilde{x}_{10} .
$$

Достижение биллиардной траекторией верхней границы означает, что координаты в момент столкновения с ней удовлетворяют уравнению

$$
\frac{\tilde{x}_{2}(t)}{\sqrt{m_{2}}}=\frac{\tilde{x}_{1}(t)}{\sqrt{m_{1}}}+\frac{L}{2} .
$$

Используя уравнения (4), легко найти время столкновения из уравнения

$$
t^{2}+\frac{2 m_{2}}{e_{2} E}\left(\frac{\widetilde{V}_{2, n}}{\sqrt{m_{2}}}-\frac{\widetilde{V}_{1, n}}{\sqrt{m_{1}}}\right) t+\frac{2 m_{2} L}{e_{2} E}=0 .
$$

Решение этого квадратного уравнения дает два возможных корня. Нужное значение времени столкновения $t_{\mathrm{c} 1}$ определяется меньшим положительным корнем:

$$
t_{\mathrm{c} 1}=\frac{m_{2}}{e_{2} E}\left\{\left(\frac{\widetilde{V}_{1, n}}{\sqrt{m_{1}}}-\frac{\widetilde{V}_{2, n}}{\sqrt{m_{2}}}\right)-\sqrt{\left(\frac{\widetilde{V}_{1, n}}{\sqrt{m_{1}}}-\frac{\widetilde{V}_{2, n}}{\sqrt{m_{2}}}\right)^{2}-\frac{2 e_{2} E L}{m_{2}}}\right\} .
$$

Чтобы столкновение с верхней границей биллиарда было возможно, должно выполняться условие

$$
\left(\frac{\widetilde{V}_{1, n}}{\sqrt{m_{1}}}-\frac{\widetilde{V}_{2, n}}{\sqrt{m_{2}}}\right)^{2} \geqslant \frac{2 e_{2} E L}{m_{2}},
$$

означающее, что кинетическая энергия относительных движений должна превышать потенциальную энергию заряженной внутренней частицы в постоянном электрическом поле. При этом значения корней квадратного уравнения действительные.

Теперь можно определить скорости оболочки и внутренней частицы непосредственно перед столкновением:

$$
\begin{aligned}
& \widetilde{V}_{2}\left(t_{\mathrm{c} 1}-0\right)=\frac{e_{2} E}{\sqrt{m_{2}}} t_{\mathrm{c} 1}+\widetilde{V}_{2, n}=\sqrt{m_{2}}\left\{\frac{\widetilde{V}_{1, n}}{\sqrt{m_{1}}}-\sqrt{\left(\frac{\widetilde{V}_{1, n}}{\sqrt{m_{1}}}-\frac{\widetilde{V}_{2, n}}{\sqrt{m_{2}}}\right)^{2}-\frac{2 e_{2} E L}{m_{2}}}\right\}, \\
& \widetilde{V}_{1}\left(t_{\mathrm{c} 1}-0\right)=\widetilde{V}_{1, n}
\end{aligned}
$$


Однако для построения искомого отображения нужно найти значения скоростей после столкновения. Для этого, используя законы сохранения импульса и энергии (разумеется, в биллиардных координатах), установим закон отражения от границы и найдем скорости $\widetilde{V}_{2, n+1}=\widetilde{V}_{2}\left(t_{\mathrm{c} 1}+0\right)$ и $\widetilde{V}_{1, n+1}=\widetilde{V}_{1}\left(t_{\mathrm{c} 1}+0\right)$ сразу после отражения от верхней границы. После простых преобразований получим

$$
\begin{aligned}
& \widetilde{V}_{2, n+1}=\frac{2 \sqrt{m_{2} m_{1}}}{m_{2}+m_{1}} \widetilde{V}_{1}\left(t_{\mathrm{c} 1}-0\right)+\frac{m_{2}-m_{1}}{m_{2}+m_{1}} \widetilde{V}_{2}\left(t_{\mathrm{c} 1}-0\right), \\
& \widetilde{V}_{1, n+1}=\frac{m_{1}-m_{2}}{m_{2}+m_{1}} \widetilde{V}_{1}\left(t_{\mathrm{c} 1}-0\right)+\frac{2 \sqrt{m_{2} m_{1}}}{m_{2}+m_{1}} \widetilde{V}_{2}\left(t_{\mathrm{c} 1}-0\right) .
\end{aligned}
$$

Подставляя значение скоростей до столкновения, получим закон преобразования скоростей при столкновении с верхней границей биллиарда:

$$
\begin{aligned}
& \widetilde{V}_{2, n+1}=\frac{\sqrt{m_{2}}}{\sqrt{m_{1}}} \widetilde{V}_{2, n}-\frac{\sqrt{m_{2}}\left(m_{2}-m_{1}\right)}{m_{2}+m_{1}} \sqrt{\left(\frac{\widetilde{V}_{1, n}}{\sqrt{m_{1}}}-\frac{\widetilde{V}_{2, n}}{\sqrt{m_{2}}}\right)^{2}-\frac{2 e_{2} E L}{m_{2}},} \\
& \widetilde{V}_{2, n+1}=\widetilde{V}_{1, n}-\frac{2 m_{2} \sqrt{m_{1}}}{m_{2}+m_{1}} \sqrt{\left(\frac{\widetilde{V}_{1, n}}{\sqrt{m_{1}}}-\frac{\widetilde{V}_{2, n}}{\sqrt{m_{2}}}\right)^{2}-\frac{2 e_{2} E L}{m_{2}}} .
\end{aligned}
$$

Полученное выше нелинейное отображение определяет преобразование скоростей $\widetilde{V}_{1, n}, \widetilde{V}_{2, n}$, которые имеют составляющие композитной частицы непосредственно после столкновения с нижней границей, в значения скоростей $\widetilde{V}_{1, n+1}, \widetilde{V}_{2, n+1}$ сразу после столкновения с верхней границей биллиарда. После этого траектория снова достигает нижней границы биллиарда. Используя закон изменения скоростей после отражения, найдем момент достижения нижней границы из условия

$$
\frac{\tilde{x}_{2}(t)}{\sqrt{m_{1}}}=\frac{\tilde{x}_{1}(t)}{\sqrt{m_{2}}}-\frac{L}{2} .
$$

Фактически повторяя предыдущие рассуждения, определим время столкновения с нижней границей:

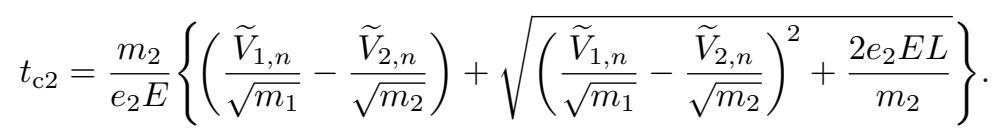

После этого, как и выше, найдем скорости $\widetilde{V}_{1, n+2}, \widetilde{V}_{2, n+2}$ после столкновения с нижней границей. Опуская аналогичные предыдущим вычисления, приведем вид этого отображения:

$$
\begin{aligned}
& \widetilde{V}_{2, n+2}=\frac{\sqrt{m_{2}}}{\sqrt{m_{1}}} \widetilde{V}_{1, n+1}+\frac{\sqrt{m_{2}}\left(m_{2}-m_{1}\right)}{m_{2}+m_{1}} \sqrt{\left(\frac{\widetilde{V}_{1, n+1}}{\sqrt{m_{1}}}-\frac{\widetilde{V}_{2, n+1}}{\sqrt{m_{2}}}\right)^{2}+\frac{2 e_{2} E L}{m_{2}}} \\
& \widetilde{V}_{1, n+2}=\widetilde{V}_{1, n+1}+\frac{2 m_{2} \sqrt{m_{1}}}{m_{2}+m_{1}} \sqrt{\left(\frac{\widetilde{V}_{1, n+1}}{\sqrt{m_{1}}}-\frac{\widetilde{V}_{2, n+1}}{\sqrt{m_{2}}}\right)^{2}+\frac{2 e_{2} E L}{m_{2}}} .
\end{aligned}
$$

Таким образом, эволюция скоростей в дискретном времени определяется последовательным применением нелинейных отображений (8) и (10). Эти отображения 
описывают изменение скоростей в режиме, когда биллиардная траектория последовательно отражается то от нижней, то от верхней границ биллиарда. Условие реализации такого режима задается неравенством (8). Физически это означает, что внутренняя частица последовательно сталкивается то с левой, то с правой границами оболочки. Времена $t_{\mathrm{c} 1}$ и $t_{\mathrm{c} 2}$ между столкновениями определяются соотношениями (5) и (9). В этом режиме ускорение оболочки происходит в результате передачи импульса от внутренней частицы при столкновении с обеими границами оболочки.

Однако такой режим не является единственно возможным. Существует режим движения, при котором внутренняя частица сталкивается только с одной стороной границы оболочки или, в биллиардной идеологии, только с одной границей биллиарда. Такой режим реализуется, если не выполнено условие (6). Тогда время столкновения с верхней границей биллиарда становится мнимым. Это означает, что частица не достигает этой границы и столкновение происходит снова с нижней границей биллиарда. Геометрический смысл этого условия обсудим детально в следующем разделе, а здесь рассмотрим, как преобразуются скорости при данном режиме.

Пусть снова на некотором шаге эволюции траектория отразилась от нижней границы. Сразу после отражения скорости внутренней частицы и оболочки равны $\widetilde{V}_{1, n}, \widetilde{V}_{2, n}$ в биллиардных переменных. Точка отражения $\left(\tilde{x}_{10}, \tilde{x}_{20}\right)$ лежит на нижней границе биллиарда: $\tilde{x}_{10} / \sqrt{m_{1}}=\tilde{x}_{20} / \sqrt{m_{2}}-L / 2$. После этого траектория, не достигая верхней границы, падает на нижнюю границу биллиарда. Другими словами, в момент столкновения с нижней границей координаты удовлетворяют уравнению

$$
\frac{\tilde{x}_{2}(t)}{\sqrt{m_{1}}}=\frac{\tilde{x}_{1}(t)}{\sqrt{m_{2}}}-\frac{L}{2} .
$$

Используя законы движения (4), легко найти из этого уравнения время столкновения:

$$
t_{\mathrm{c}}=\frac{2 m_{2}}{E e_{2}}\left(\frac{\widetilde{V}_{1, n}}{\sqrt{m_{1}}}-\frac{\widetilde{V}_{2, n}}{\sqrt{m_{2}}}\right) .
$$

После этого легко вычислить скорости оболочки и частицы в момент столкновения:

$$
\widetilde{V}_{2}\left(t_{\mathrm{c}}-0\right)=\frac{E e_{2}}{\sqrt{m_{2}}} t_{\mathrm{c}}+\widetilde{V}_{2, n}=2 \frac{\sqrt{m_{2}}}{\sqrt{m_{1}}} \widetilde{V}_{1, n}-\widetilde{V}_{2, n}, \quad \widetilde{V}_{1}\left(t_{\mathrm{c}}-0\right)=\widetilde{V}_{1, n} .
$$

Используя законы (7) преобразования скоростей при столкновении с границей биллиарда, перейдем к скоростям $\widetilde{V}_{2, n+1}=\widetilde{V}_{2}\left(t_{\mathrm{c}}+0\right)$ и $\widetilde{V}_{1, n+1}=\widetilde{V}_{1}\left(t_{\mathrm{c}}+0\right)$ сразу после столкновения с нижней границей:

$$
\begin{aligned}
\widetilde{V}_{2, n+1} & =\frac{2 m_{2} \sqrt{m_{2}}}{\sqrt{m_{1}}\left(m_{2}+m_{1}\right)} \widetilde{V}_{1, n}+\frac{m_{1}-m_{2}}{m_{2}+m_{1}} \widetilde{V}_{2, n}, \\
\widetilde{V}_{1, n+1} & =\frac{3 m_{2}+m_{1}}{m_{2}+m_{1}} \widetilde{V}_{1, n}-\frac{2 \sqrt{m_{2} m_{1}}}{m_{2}+m_{1}} \widetilde{V}_{2, n} .
\end{aligned}
$$

Так как в рассматриваемом режиме столкновения всегда происходят с нижней границей биллиарда, это отображение полностью описывает изменение скоростей в дискретном времени. Интересно отметить, что в отображение (11) в явном виде не входит электрическое поле. Разумеется, это не означает, что влияние электрического поля отсутствует: электрическое поле сказывается на шаге дискретизации. 
После вывода законов эволюции в биллиардных координатах можно вернуться и к исходным координатам. Нетрудно записать отображения (8) и (10), соответствующие режиму столкновений с обеими стенками, в исходных координатах:

$$
\begin{aligned}
& V_{2, n+1}=V_{1, n}-\frac{m_{2}-m_{1}}{m_{2}+m_{1}} \sqrt{\left(V_{1, n}-V_{2, n}\right)^{2}-\frac{2 e_{2} E L}{m_{2}}}, \\
& V_{1, n+1}=V_{1, n}-\frac{2 m_{2}}{m_{2}+m_{1}} \sqrt{\left(V_{1, n}-V_{2, n}\right)^{2}-\frac{2 e_{2} E L}{m_{2}}}, \\
& V_{2, n+2}=V_{1, n+1}+\frac{m_{2}-m_{1}}{m_{2}+m_{1}} \sqrt{\left(V_{1, n+1}-V_{2, n+1}\right)^{2}+\frac{2 e_{2} E L}{m_{2}}}, \\
& V_{1, n+2}=V_{1, n+1}+\frac{2 m_{2}}{m_{2}+m_{1}} \sqrt{\left(V_{1, n+1}-V_{2, n+1}\right)^{2}+\frac{2 e_{2} E L}{m_{2}}} .
\end{aligned}
$$

Отображения, соответствующие режиму отражения только от одной границы биллиарда, в этих координатах принимают вид

$$
\begin{aligned}
V_{2, n+1} & =\frac{2 m_{2}}{m_{2}+m_{1}} V_{1, n}-\frac{m_{2}-m_{1}}{m_{2}+m_{1}} V_{2, n}, \\
V_{1, n+1} & =\frac{3 m_{2}+m_{1}}{m_{2}+m_{1}} V_{1, n}-\frac{2 m_{2}}{m_{2}+m_{1}} V_{2, n} .
\end{aligned}
$$

Еще одна форма этих отображений, удобная для интерпретации поведения композитной частицы, связана с переходом к физически важным координатам, соответствующим скорости движения центра масс $U_{n}=\left(m_{2} V_{2, n}+m_{1} V_{1, n}\right) /\left(m_{2}+m_{1}\right)$ композитной частицы и относительной скорости $R_{n}=V_{1, n}-V_{2, n}$. Отображения (12) в этих переменных принимают более простой вид:

$$
\begin{gathered}
R_{n+1}=-\sqrt{R_{n}^{2}-\frac{2 e_{2} E L}{m_{2}}}, \quad R_{n+2}=\sqrt{R_{n+1}^{2}+\frac{2 e_{2} E L}{m_{2}}}, \\
U_{n+1}=U_{n}+\frac{m_{2}}{m_{2}+m_{1}}\left(R_{n}+R_{n+1}\right), \\
U_{n+2}=U_{n+1}+\frac{m_{2}}{m_{2}+m_{1}}\left(R_{n+1}+R_{n+2}\right) .
\end{gathered}
$$

Легко заметить два важных свойства этих отображений. Прежде всего, поведение относительных скоростей не зависит от скорости центра масс. Кроме этого, можно заметить, что композиция $R_{n+2} \circ R_{n+1}=I$, где $I$ - тождественное отображение. Это означает, что величина относительной скорости после отражения от фиксированной стенки не меняется. Другими словами, если связывать относительную скорость с тепловой скоростью внутренних степеней свободы, то можно сказать, что нагрева внутренней степени свободы такой композитной частицы не происходит. Увеличение скорости центра масс пропорционально сумме $R_{n}+R_{n+1}$ относительных скоростей при столкновении с противоположными концами оболочки. В этом смысле последнее из отображений (14) лишнее и полностью совпадает с предшествующим ему. Таким образом, отображения (14) переходят в

$$
R_{n+1}=R_{n}, \quad U_{n+1}=U_{n}+\frac{2 m_{2}}{m_{2}+m_{1}} R_{n} .
$$



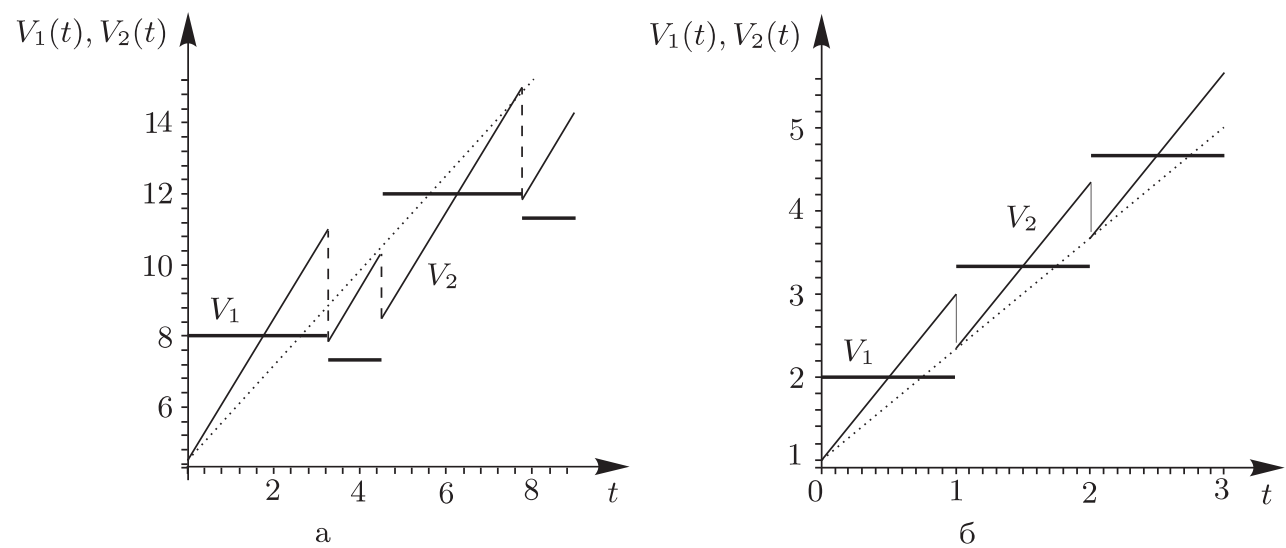

Рис. 3. Типичный характер изменения скорости оболочки и внутренней частицы в режиме, когда столкновения происходят с обеими границами оболочки (а), и в режиме, когда внутренняя частица сталкивается только с одной границей оболочки (б). Жирной линией показано изменение скорости оболочки, тонкой - скорости внутренней частицы. Пунктирная линия приведена для сравнения, она показывает ускорение бесструктурной частицы с зарядом $e_{2}$, массой $m_{2}+m_{1}$ и начальной скоростью $V_{1}$ в таком же электрическом поле.

Поведение композитной частицы в случае, когда столкновения происходят только с одной из границ оболочки, описывается особенно просто. Действительно, как следует из отображений (15), относительная скорость в моменты столкновений оболочки и внутренней частицы не меняется. С физической точки зрения это означает отсутствие "нагрева" внутренней степени свободы. Скорость центра масс монотонно нарастает при каждом столкновении, увеличиваясь на постоянную величину, пропорциональную относительной скорости. Интересно отметить, что эффект наличия внутренних степеней свободы проявляется как своеобразная дискретность изменения скорости движения композитной частицы. Изменение скорости происходит на "квант" скорости, который определяется отношением масс и начальной относительной скоростью. Величина этого "кванта" позволяет найти одну из этих величин по наблюдаемому изменению скорости композитной частицы. При этом измерение времен периода смены скорости композитной частицы также несет информацию о массе внутренней частицы и относительных скоростях оболочки и внутренней частицы. Однако по этим данным можно установить величину заряда внутренней частицы, но не ее массу.

Зависимость от времени скоростей внутренней частицы и оболочки легко восстанавливается по данным отображений. На рис. 3 приведен качественный вид изменения скоростей в этих двух режимах.

Итак, мы полностью описали эволюцию композитной частицы с одной внутренней степенью свободы при условии, что заряжена только внутренняя частица.

Обратимся теперь к случаю общего положения композитной частицы с одной степенью свободы. Пусть внутренняя частица имеет заряд $e_{2}$, а оболочка - заряд $e_{1}$. 
Разумеется, в этом случае важна не только величина заряда, но и характер его распределения по оболочке. Сначала будем предполагать равномерное распределение заряда по оболочке, что приводит к отсутствию кулоновского взаимодействия внутренней частицы с оболочкой. Как и ранее, мы считаем, что массы внутренней частицы и оболочки равны $m_{2}$ и $m_{1}$. Конфигурационное пространство в биллиардных координатах есть полоса, показанная на рис. 2. Направление ее оси определяется единичным вектором $\vec{\tau}$, касательным к границе. В биллиардных координатах этот вектор имеет вид

$$
\vec{\tau}=\frac{1}{\sqrt{m_{1}+m_{2}}}\left(\sqrt{m_{1}}, \sqrt{m_{2}}\right) .
$$

Введем также вектор нормали $\vec{n}$ (см. рис. 2) к границе конфигурационного пространства,

$$
\vec{n}=\frac{1}{\sqrt{m_{1}+m_{2}}}\left(-\sqrt{m_{2}}, \sqrt{m_{1}}\right),
$$

его направление выбрано от нижней границы внутрь полосы.

Уравнения движения компонент композитной частицы под действием постоянного поля до столкновений имеют вид

$$
\sqrt{m_{1}} \ddot{\tilde{x}}_{1}=e_{1} E, \quad \sqrt{m_{2}} \ddot{\tilde{x}}_{2}=e_{2} E .
$$

Эти уравнения можно интерпретировать как уравнения движения эффективной частицы в полосе с постоянным ускорением

$$
\vec{a}=\left(\frac{e_{1}}{\sqrt{m_{1}}} E, \frac{e_{2}}{\sqrt{m_{2}}} E\right) .
$$

Такое ускорение создается эффективным электрическим полем $\vec{a}$, действующим на частицу с единичной массой и единичным зарядом. Направление этого эффективного электрического поля определяется массами и зарядами внутренней частицы и оболочки. Столкновение с границей биллиарда в соответствии с законами сохранения энергии и импульса происходит, как в обычном биллиарде.

Таким образом, и в этом случае движение композитной частицы сводится к движению одной эффективной заряженной точечной частицы в биллиарде в форме полосы под действием постоянного электрического поля. Далее удобно разделить движение эффективной частицы в полосе на две компоненты: продольную $\vec{x}_{\|}$вдоль оси полосы или вектора $\vec{\tau}$ и поперечную $\vec{x}_{\perp}$ по направлению нормали $\vec{n}$. Соответственно по этим направлениям удобно разложить и вектор скорости, $\overrightarrow{\vec{V}}=\overrightarrow{\vec{V}}_{\|}+\overrightarrow{\vec{V}}_{\perp}$, и поле ускорения, $\vec{a}=\vec{a}_{\|}+\vec{a}_{\perp}$. Движение в продольном и поперечном направлениях осуществляется независимо, поэтому движение в продольном направлении на всех временах эволюции - это просто равноускоренное движение под действием продольного электрического поля $\vec{a}_{\|}$. Другими словами, изменение продольной скорости определяется соотношением $\widetilde{V}_{\|}=a_{\|} t+\widetilde{V}_{\| 0}$. Здесь $\widetilde{V}_{\| 0}-$ начальная скорость продольных движений, которая связана с исходными скоростями соотношением $\widetilde{V}_{\| 0}=\left(\sqrt{m_{1}} \widetilde{V}_{01}+\sqrt{m_{2}} \widetilde{V}_{02}\right) / \sqrt{m_{1}+m_{2}}$.

Величина продольного ускорения легко находится путем разложения вектора $\vec{a}$ по направлению $\vec{\tau}$ и равна $a_{\|}=E\left(e_{1}+e_{2}\right) / \sqrt{m_{1}+m_{2}}$. Физический смысл продольной 
скорости установить достаточно просто. Легко заметить, что эта скорость отличается от скорости центра масс исходной системы только постоянным множителем. Действительно,

$$
\widetilde{V}_{\|}=\frac{1}{\sqrt{m_{1}+m_{2}}}\left(\sqrt{m_{1}} \widetilde{V}_{1}+\sqrt{m_{2}} \widetilde{V}_{2}\right)=\sqrt{m_{1}+m_{2}} \frac{m_{1} V_{1}+m_{2} V_{2}}{m_{1}+m_{2}} .
$$

Таким образом, мы получили описание продольной компоненты скорости. Изменение продольных координат до столкновения с границей биллиарда, соответственно, определяется уравнением

$$
x_{\|}=a_{\|} \frac{t^{2}}{2}+\widetilde{V}_{\| 0} t+\tilde{x}_{\| 0},
$$

где $x_{\| 0}=\overrightarrow{\tilde{x}}_{0} \cdot \vec{\tau}$ - начальное положение эффективной частицы в продольном направлении.

В случае равенства нулю поперечного ускорения

$$
\vec{a}_{\perp}=\frac{E}{\sqrt{m_{1}+m_{2}}} \frac{m_{1} e_{2}-m_{2} e_{1}}{\sqrt{m_{1} m_{2}}} \vec{n},
$$

т. е. при специальном соотношении $m_{1} e_{2}=m_{2} e_{1}$ масс и зарядов и равенстве нулю поперечной начальной скорости реализуется простейший режим движения эффективной частицы - движение параллельно границе биллиарда без столкновений с ней. Для композитной частицы это означает согласованное равноускоренное движение внутренней частицы и оболочки без столкновений друг с другом. Ясно, что такой режим разрушается при появлении начальной поперечной скорости или нарушении равенства $m_{1} e_{2}=m_{2} e_{1}$. При отличной от нуля начальной поперечной скорости реализуется режим периодического поперечного движения с постоянной скоростью между столкновениями с границей биллиарда и равноускоренное движение в продольном направлении.

Теперь обратимся к более типичным режимам движения. Проанализируем поперечную компоненту движения эффективной частицы. Предположим, что в начальный момент эффективная частица расположена на нижней границе биллиарда, т. е. $\tilde{x}_{1} / \sqrt{m_{1}}-\tilde{x}_{2} / \sqrt{m_{2}}=L / 2$. При наличии столкновений с нижней границей в качестве начального положения можно выбрать точку столкновения с границей. Соответствующую поперечную координату обозначим как $\tilde{x}_{\perp n}$, снабдив ее индексом $n$. Причина этого станет ясна далее. Разумеется, частица обладает и некоторой начальной поперечной скоростью $\widetilde{V}_{\perp n}$, направленной по нормали $\vec{n}$. Эту скорость также снабдим индексом $n$. Физический смысл поперечной скорости можно установить, возвращаясь к исходным скоростям:

$$
V_{\perp}=\frac{1}{\sqrt{m_{1}+m_{2}}}\left(\sqrt{m_{1}} \widetilde{V}_{2}-\sqrt{m_{2}} \widetilde{V}_{1}\right)=\frac{\sqrt{m_{1} m_{2}}}{\sqrt{m_{1}+m_{2}}}\left(V_{2}-V_{1}\right) .
$$

Видно, что поперечная скорость связана с относительной скоростью компонент. Изменение поперечной скорости до достижения верхней границы биллиарда определяется действием на нее поперечного постоянного поля и описывается формулой $\widetilde{V}_{\perp}=a_{\perp} t+\widetilde{V}_{\perp n}$. Это уравнение справедливо вплоть до момента достижения эффективной частицей границы биллиарда, при $0 \leqslant t \leqslant t_{\mathrm{c} 1}$. 
Вычислим время столкновения с верхней стенкой биллиарда. Учитывая начальное положение частицы, заключаем, что до столкновения с верхней стенкой частица должна преодолеть расстояние $\widetilde{L}=L \sqrt{m_{1} m_{2}} / \sqrt{m_{1}+m_{2}}$ между границами биллиарда. Таким образом, используя закон изменения поперечных координат, получим уравнение для определения времени столкновения с верхней границей:

$$
\widetilde{L}=a_{\perp} \frac{t_{\mathrm{c} 1}^{2}}{2}+\widetilde{V}_{\perp n} t_{\mathrm{c} 1}
$$

Действительное решение этого квадратного уравнения существует, если выполняется неравенство

$$
\frac{\widetilde{V}_{\perp n}^{2}}{a_{\perp}^{2}}+\frac{2 \widetilde{L}}{a_{\perp}} \geqslant 0 .
$$

Физически только при этом условии происходит столкновение с верхней стенкой. При выполнении обратного неравенства частица под действием поля разворачивается, не достигая верхней границы, и сталкивается с нижней стенкой. В этом случае уравнение для времени столкновения определяется условием возврата к начальному поперечному положению и имеет вид

$$
a_{\perp} \frac{t_{\mathrm{c} 1}^{2}}{2}+\widetilde{V}_{\perp n} t_{\mathrm{c} 1}=0
$$

Предполагая время столкновений отличным от нуля, находим $t_{\mathrm{c} 1}=-2 \widetilde{V}_{\perp n} / a_{\perp}$. Следовательно, в момент столкновения скорость частицы $\widetilde{V}_{\perp}\left(t_{\mathrm{c} 1}-0\right)=a_{\perp} t_{\mathrm{c} 1}+\widetilde{V}_{\perp n}$. При столкновении согласно закону отражения в биллиарде меняет знак поперечная скорость, а продольная сохраняется. Тогда сразу после столкновения поперечная скорость равна

$$
\widetilde{V}_{\perp, n+1}=-\left(a_{\perp} t_{\mathrm{c} 1}+\widetilde{V}_{\perp n}\right)=-\left(a_{\perp}\left(-\frac{2 \widetilde{V}_{\perp n}}{a_{\perp}}\right)+\widetilde{V}_{\perp n}\right)=\widetilde{V}_{\perp n} .
$$

Далее цикл повторяется. Таким образом, отображение, определяющее эволюцию поперечной скорости при выполнении неравенства $\widetilde{V}_{\perp n}^{2} / a_{\perp}^{2}+2 \widetilde{L} / a_{\perp}<0$ и при начальной скорости, направленной к верхней границе, имеет исключительно простой вид:

$$
\widetilde{V}_{\perp, n+1}=\widetilde{V}_{\perp n}
$$

В этом отображении $\widetilde{V}_{\perp n}$ имеет смысл скорости сразу после $n$-го столкновения со стенкой. Остается добавить отображение для изменения продольной компоненты во времена столкновений. Это отображение легко получить из равноускоренного характера изменения продольной скорости:

$$
\widetilde{V}_{\|, n+1}=\widetilde{V}_{\|, n+1}-2 \widetilde{V}_{\perp n} \frac{a_{\|}}{a_{\perp}} .
$$

Отображения $(17),(18)$ описывают динамику композитной частицы в режиме столкновения эффективной частицы только с одной стенкой. 
Вернемся теперь к случаю, когда эффективная частица достигает верхней границы биллиарда. В этом случае ее поперечная скорость непосредственно перед столкновением определяется соотношением $a_{\perp} t_{\mathrm{c} 1}\left(\widetilde{V}_{\perp n}\right)+\widetilde{V}_{\perp n}$, где

$$
t_{\mathrm{c} 1}\left(\widetilde{V}_{\perp n}\right)=\min _{t>0}\left(-\frac{V_{\perp n}}{a_{\perp}} \pm \sqrt{\frac{V_{\perp n}}{a_{\perp}^{2}}+\frac{2 \widetilde{L}}{a_{\perp}}}\right)
$$

(символ $\min _{t>0}$ означает выбор из двух положительных корней минимального, если таких решений два). После отражения от верхней стенки поперечная скорость меняет знак и соответственно

$$
\widetilde{V}_{\perp, n+1}=-\left(a_{\perp} t_{\mathrm{c} 1}\left(\widetilde{V}_{\perp n}\right)+\widetilde{V}_{\perp n}\right) .
$$

Далее частица направляется к нижней границе и достигает ее в момент времени $t_{\mathrm{c} 2}$, который определяется уравнением

$$
a_{\perp} \frac{t_{\mathrm{c} 2}^{2}}{2}+V_{\perp, n+1} t_{\mathrm{c} 2}+\widetilde{L}=0 .
$$

Это квадратное уравнение имеет действительное решение, если

$$
\frac{\widetilde{V}_{\perp, n+1}^{2}}{a_{\perp}^{2}}-\frac{2 \widetilde{L}}{a_{\perp}} \geqslant 0
$$

(разумеется, интерес представляют положительные решения). Если это неравенство не выполнено, то частица не достигает нижней стенки и далее будет сталкиваться только с верхней границей биллиарда. Другими словами, происходит выход на описанный ранее режим. Отличие заключается только в том, что столкновения частицы будут происходить с верхней, а не нижней стенкой. Если неравенство (20) выполняется, то легко найти время столкновения с нижней стенкой:

$$
t_{\mathrm{c} 2}\left(\widetilde{V}_{\perp, n+1}\right)=\min _{t>0}\left(-\frac{V_{\perp, n+1}}{a_{\perp}} \pm \sqrt{\frac{V_{\perp, n+1}}{a_{\perp}^{2}}-\frac{2 \widetilde{L}}{a_{\perp}}}\right) .
$$

Как и ранее, поперечная скорость непосредственно перед столкновением рассчитывается без труда, она равна $a_{\perp} t_{\mathrm{c} 2}\left(\widetilde{V}_{\perp, n+1}\right)+V_{\perp, n+1}$. Учитывая изменение знака поперечной скорости после столкновения с границей биллиарда, запишем отображение, определяющее скорость после отражения от нижней границы:

$$
\widetilde{V}_{\perp, n+2}=-\left(a_{\perp} t_{\mathrm{c} 2}\left(\widetilde{V}_{\perp, n+1}\right)+V_{\perp, n+1}\right) .
$$

Скорость после следующего отражения от границы биллиарда определяется отображением (21). Таким образом, отображения, определяющие изменение поперечной скорости частицы, задаются соотношениями (19), (21). Подставляя соответствующие ветви времен столкновений, получим явный вид динамических отображений для поперечных скоростей:

$$
\widetilde{V}_{\perp, n+1}=-\sqrt{\widetilde{V}_{\perp n}^{2}+2 \widetilde{L} a_{\perp}}, \quad \widetilde{V}_{\perp, n+2}=\sqrt{\widetilde{V}_{\perp, n+1}^{2}-2 \widetilde{L} a_{\perp}} .
$$


Легко заметить, что $\widetilde{V}_{\perp, n+2}=\widetilde{V}_{\perp n}$. Это означает, что поперечная скорость меняется периодически.

Разумеется, если интересоваться изменением всех скоростей композитной частицы, то полная система отображений должна быть дополнена отображениями, определяющими эволюцию продольной скорости. Тогда полная система отображений принимает вид

$$
\begin{gathered}
\widetilde{V}_{\perp, n+1}=-\sqrt{\widetilde{V}_{\perp n}^{2}+2 \widetilde{L}_{\perp}}, \quad \widetilde{V}_{\perp, n+2}=\sqrt{\widetilde{V}_{\perp, n+1}^{2}-2 \widetilde{L}_{\perp}}, \\
\widetilde{V}_{\|, n+1}=\frac{a_{\|}}{a_{\perp}}\left(\sqrt{\widetilde{V}_{\perp n}^{2}+2 \widetilde{L} a_{\perp}}-\widetilde{V}_{\perp n}\right)+\widetilde{V}_{\| n}, \\
\widetilde{V}_{\|, n+2}=-\frac{a_{\|}}{a_{\perp}}\left(\sqrt{\widetilde{V}_{\perp, n+1}^{2}-2 \widetilde{L} a_{\perp}}+\widetilde{V}_{\perp, n+1}\right)+\widetilde{V}_{\|, n+1} .
\end{gathered}
$$

Замечая, что отображения для $n+2$ и $n+1$ отличаются только знаками, можно привести динамические отображения к более простой форме двух отображений:

$$
\begin{aligned}
& \widetilde{V}_{\perp, n+1}=-\frac{\widetilde{V}_{\perp n}}{\left|\widetilde{V}_{\perp n}\right|} \sqrt{\widetilde{V}_{\perp n}^{2}+2 \widetilde{L} a_{\perp} \frac{\widetilde{V}_{\perp n}}{\left|\widetilde{V}_{\perp n}\right|}}, \\
& \widetilde{V}_{\|, n+1}=-\frac{a_{\|}}{a_{\perp}}\left(\widetilde{V}_{\perp, n+1}-\frac{\widetilde{V}_{\perp, n+1}}{\left|\widetilde{V}_{\perp, n+1}\right|} \widetilde{V}_{\perp n}\right)+\widetilde{V}_{\| n} .
\end{aligned}
$$

Это динамическое отображение полностью определяет эволюцию скоростей в случае общего положения при $\widetilde{V}_{\perp n} \neq 0$. Все режимы движения сводятся к монотонному росту продольной скорости и периодическому изменению поперечной скорости. Своеобразная дискретность в изменении скорости композитной частицы сохраняется и в этом случае.

Динамические отображения, определяющие поведение внутренней степени свободы и оболочки, легко получить из этих отображений, учитывая, что $\widetilde{V}_{\perp n}$ и $\widetilde{V}_{\| n}$ являются линейной комбинацией исходных скоростей $V_{1}$ и $V_{2}$. Исходные скорости выражаются через продольную и поперечную скорости согласно уравнениям

$$
V_{1}=\frac{1}{\sqrt{m_{1}+m_{2}}}\left(\widetilde{V}_{\|}-\frac{\sqrt{m_{2}}}{\sqrt{m_{1}}} \widetilde{V}_{\perp}\right), \quad V_{2}=\frac{1}{\sqrt{m_{1}+m_{2}}}\left(\widetilde{V}_{\|}+\frac{\sqrt{m_{1}}}{\sqrt{m_{2}}} \widetilde{V}_{\perp}\right) .
$$

Используя эти соотношения, нетрудно вывести точные динамические отображения и в терминах исходных скоростей. Таким образом, поведение заряженной композитной частицы с одной степенью свободы в постоянном электрическом поле полностью описывается простыми отображениями.

Из общего случая интересно выделить специальный случай нейтральной композитной частицы: $e_{1}+e_{2}=0$. При этих условиях исчезает продольная компонента эффективного электрического поля, $a_{\|}=0$. Тогда продольное движение происходит с сохраняющейся продольной скоростью: $\widetilde{V}_{\|, n+2}=\widetilde{V}_{\|, n+1}=\widetilde{V}_{\| n}$. Поэтому динамическое отображение значительно упрощается и сводится только к отображению для поперечной скорости. Частный случай $\widetilde{V}_{\| n}=0$ этого режима соответствует неподвижной композитной частице (ее центр инерции покоится) с периодически изменяющимися скоростями компонент. 

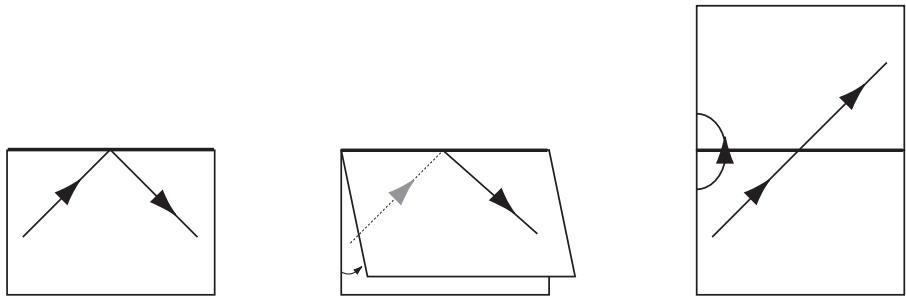

Рис. 4. Прием спрямления траекторий, предложенный Шварцем, для обычных биллиардов. Жирной линией отмечена граница биллиарда, на которую падает луч.

Еще один случай, когда заряд оболочки сосредоточен на одной из ее граней, является наиболее сложным, и мы рассмотрим его ниже после обсуждения обобщения принципа Шварца.

\section{4. “СПРЯМЛЕНИЕ" ТРАЕКТОРИЙ}

При изучении движения лучей в биллиардах один из наиболее эффективных и широко используемых приемов был предложен Шварцем. Он основан на идее спрямления биллиардной траектории [10] или на переходе от движения в биллиарде к свободному движению луча в пространстве без границ (см. рис. 4). Данную идеологию удобно использовать и при обсуждении движения композитной частицы в постоянном электрическом поле, однако для этого недостаточно отразить биллиард относительно границы, на которую падает траектория. Кроме этого, нужно также обобщить прием Шварца и изменить подходящим образом вид электрического поля в отраженном биллиарде. Таким образом, правильное продолжение траектории в отраженном биллиарде предполагает и правильное изменение потенциала электрического поля. Легко понять, что вместе с отражением биллиарда относительно границы, на которую падает заряженная частица, нужно отразить и электрическое поле (см. рис. 5). В результате появляется возможность ввести общий потенциал, движение в котором и будет описывать траекторию уже во всем пространстве без отражений от границ полосы. Электрическое поле этого потенциала будет скачкообразно меняться при переходе из полосы в полосу (см. рис. 6). Так осуществляется переход к свободному движению в определенном поле. Следует подчеркнуть, что подобное обобщение принципа Шварца исключительно полезно для решения широкого круга физических задач, связанных с биллиардами во внешних полях.

Для решения рассматриваемой задачи предварительно удобно перейти к новой системе координат, в которой границы биллиарда параллельны одной из осей координат. Такой переход не является принципиальным, но упрощает последующие вычисления. Напомним, что угол $\alpha$, под которым наклонена полоса (см. рис. 2), определяется отношением масс:

$$
\operatorname{tg} \alpha=\sqrt{\frac{m_{2}}{m_{1}}}, \quad \cos \alpha=\sqrt{\frac{m_{1}}{m_{1}+m_{2}}}, \quad \sin \alpha=\sqrt{\frac{m_{2}}{m_{1}+m_{2}}} .
$$




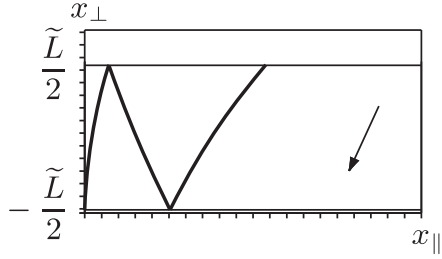

a

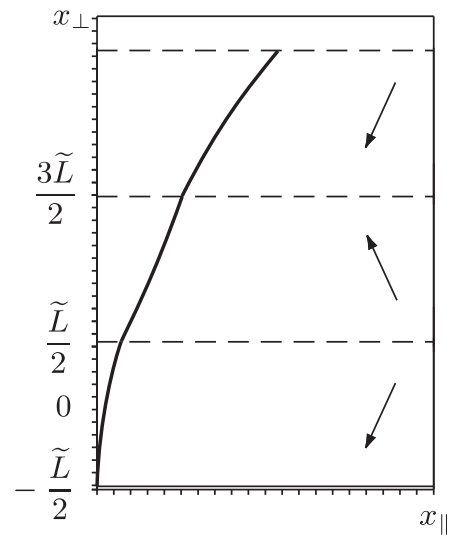

б

Рис. 5. Биллиард в выбранной системе координат $\left(x_{\|}, x_{\perp}\right)$ и несколько сегментов траектории (а); результат спрямления этой траектории (б). Хорошо заметна смена характера движения из-за изменения направления вертикальной компоненты электрического поля.

Перейдем к новой системе координат, повернутой на угол $-\alpha$ относительно исходной:

$$
x_{\|}=\tilde{x}_{1} \cos \alpha+\tilde{x}_{2} \sin \alpha, \quad x_{\perp}=-\tilde{x}_{1} \sin \alpha+\tilde{x}_{2} \cos \alpha .
$$

Другими словами, перейдем к продольным и поперечным к полосе координатам.

Запишем уравнения движения (16) в новых координатах $\left(x_{\|}, x_{\perp}\right)$. Начнем со случая, когда однородно заряжена оболочка и одна из внутренних частиц, в этом случае

$$
\begin{aligned}
& \ddot{x}_{\|}=\frac{e_{1}+e_{2}}{\sqrt{m_{1}+m_{2}}} E=\frac{e}{\sqrt{m_{1}+m_{2}}} E_{\|}, \\
& \ddot{x}_{\perp}=\frac{e_{2} m_{1}-e_{1} m_{2}}{\sqrt{m_{1} m_{2}\left(m_{1}+m_{2}\right)}} E=\frac{e}{\sqrt{m_{1}+m_{2}}} E_{\perp} .
\end{aligned}
$$

Обратим внимание, что в этой системе координат между столкновениями с границей биллиарда эффективная частица движется как точечная частица с массой $\sqrt{m_{1}+m_{2}}$ и зарядом $e$ в постоянном электрическом поле

$$
\vec{E}=\left(E_{\|}, E_{\perp}\right)=\left(\frac{e_{1}+e_{2}}{e} E, \frac{e_{2} m_{1}-e_{1} m_{2}}{e \sqrt{m_{1} m_{2}}} E\right) .
$$

Разумеется, без потери общности можно положить величину заряда $e=1$. В координатах (24) конфигурационное пространство устроено исключительно просто (см. рис. 5). На рисунке введено обозначение $\widetilde{L}=L \sqrt{m_{1} m_{2} /\left(m_{1}+m_{2}\right)}$ для ширины полосы.

Теперь достаточно очевидно, что, перекладывая полосу, как и в обычном биллиарде, нужно одновременно изменить электрическое поле. Ясно, что в выбранной системе координат при переходе из полосы в полосу вертикальная составляющая 


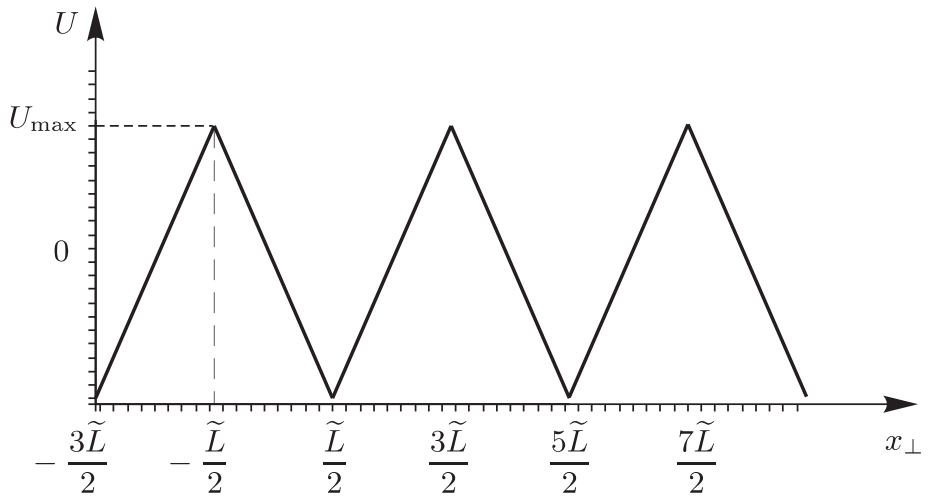

Рис. 6. Характерный вид потенциала электрического поля при использовании обобщенного спрямления траекторий. Направление электрического поля выбрано вдоль оси $\tilde{x}_{\perp}\left(e E_{\perp}>0\right)$.

поля должна менять знак, а горизонтальная компонента поля не меняется. В координатах (24) уравнение движения вдоль оси $x_{\|}$не ограничено границами и может быть проинтегрировано глобально в силу независимости от поперечных движений:

$$
\dot{x}_{\|}=\frac{e}{\sqrt{m_{1}+m_{2}}} E_{\|} t+V_{0 \|}, \quad x_{\|}(t)=\frac{e}{\sqrt{m_{1}+m_{2}}} E_{\|} \frac{t^{2}}{2}+V_{0 \|} t+x_{0 \|} .
$$

Здесь $V_{0 \|}$ и $x_{0 \|}$ - начальные продольные скорость и положение точечной частицы. Таким образом, горизонтальная, или продольная, компонента скорости и изменение координаты $x_{\|}(t)$ соответствуют движению с постоянным ускорением, которое определяется величиной эффективного продольного электрического поля.

Нетривиальным изменениям при спрямлении подвергаются только вертикальные компоненты скорости и координаты. Фактически мы можем описывать движение, в точности как движение заряженной частицы в пилообразном потенциале (см. рис. 6). Возвращаясь к уравнениям (25), установим вид эффективного потенциала $U\left(x_{\perp}\right)$, сравнивая его с уравнением $\ddot{x}_{\perp}=-\partial U\left(x_{\perp}\right) / \partial x_{\perp}$. Здесь $U\left(x_{\perp}\right)-$ периодический потенциал эффективного поперечного электрического поля.

Используя приведенное выше уравнение, легко найти вид потенциала на интервале значений $-\widetilde{L} / 2 \leqslant x_{\perp} \leqslant \widetilde{L} / 2$. После этого, меняя направление поперечного электрического поля на противоположное при каждом отражении полосы относительно границы, установим вид глобального потенциала (см. рис. 6):

$$
U\left(x_{\perp}\right)=(-1)^{n+1} \frac{e E_{\perp}}{\sqrt{m_{1}+m_{2}}}\left(x_{\perp}-\widetilde{L} n\right), \quad\left(n-\frac{1}{2}\right) \widetilde{L}<x_{\perp} \leqslant\left(n+\frac{1}{2}\right) \widetilde{L},
$$

где $n$ - целое число.

Таким образом, задача о поперечном движении в таком биллиарде сводится к движению заряженной частицы в безграничном пространстве, но в периодическом потенциале пилообразного вида, как на рис. 6. Классификация типов возможных движений в подобных потенциалах общеизвестна. В них могут существовать только 

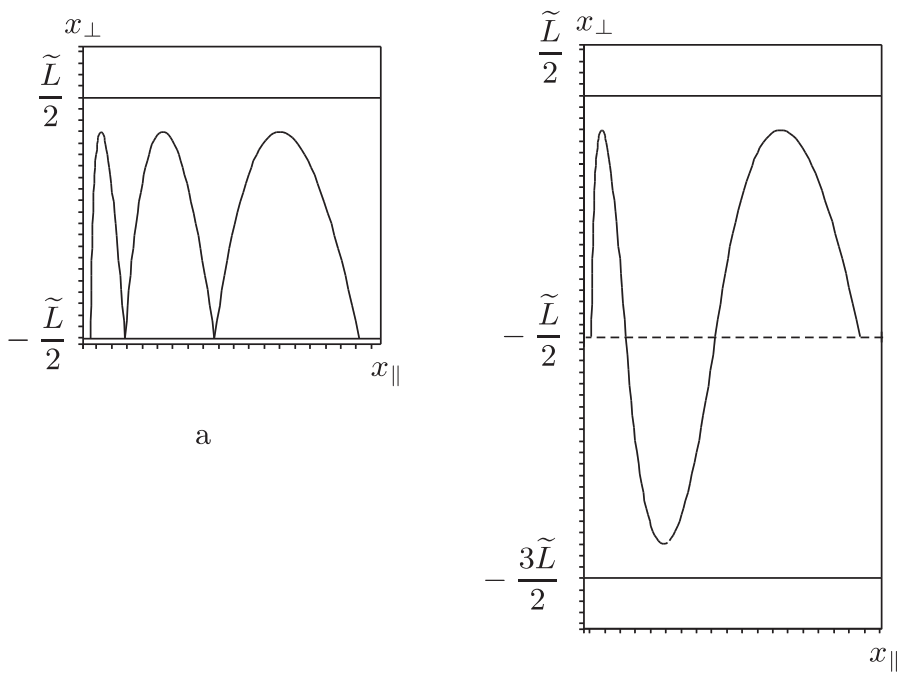

б

Рис. 7. Траектория в биллиарде, соответствующая захваченному режиму по направлению оси $x_{\perp}(\mathrm{a})$ и эта же траектория после спрямления (б).

два типа движений, соответствующие пролетным и захваченным движениям. Из-за наличия излома в максимуме потенциала кинковые решения не определены. При начальной поперечной энергии $W_{\perp}<U_{\max }$ реализуется режим захваченных движений, а при $W_{\perp}>U_{\max }$ - пролетный режим. Величина максимального значения потенциала определяется соотношением

$$
U_{\max }=\left|\frac{e E_{\perp} \widetilde{L}}{2 \sqrt{m_{1}+m_{2}}}\right| .
$$

В обоих типах режимов поперечная скорость изменяется периодически.

Также легко построить фазовый портрет поперечных движений, используя закон сохранения

$$
\frac{1}{2} \dot{x}_{\perp}^{2}+U\left(x_{\perp}\right)=W_{\perp}=\text { const. }
$$

Разумеется, используя это уравнение, можно найти как точные решения, так и период соответствующих движений. Так, периоды движения в пролетном и захваченном режимах определяются соотношениями

$$
\begin{aligned}
& T=4 \frac{\sqrt{m_{1}+m_{2}}}{e E_{\perp}}\left\{\sqrt{W_{\perp}+\frac{e E_{\perp} \widetilde{L}}{2 \sqrt{m_{1}+m_{2}}}}-\sqrt{W_{\perp}-\frac{e E_{\perp} \widetilde{L}}{2 \sqrt{m_{1}+m_{2}}}}\right\}, \\
& T=4 \frac{\sqrt{m_{1}+m_{2}}}{e E_{\perp}} \sqrt{W_{\perp}+\frac{e E_{\perp} \widetilde{L}}{2 \sqrt{m_{1}+m_{2}}}}
\end{aligned}
$$

соответственно. 
Пример пролетной траектории приведен на рис. 5, а захваченной - на рис. 7. Переход от безграничной к исходной задаче в биллиарде сводится к складыванию траектории. Например, траектория, показанная на рис. 5а, после спрямления принимает вид, показанный на рис. 5б. Разумеется, можно ввести потенциал эффективного электрического поля и во всем пространстве, не ограничиваясь поперечной компонентой. Глобально потенциальная энергия есть сумма потенциала (27) и потенциальной энергии $U_{\|}\left(x_{\|}\right)=-e E / \sqrt{m_{1}+m_{2}} \cdot x_{\|}$, зависящей от продольной координаты. Таким образом, движение композитной частицы с одной внутренней степенью свободы в постоянном электрическом поле сводится к движению одной точечной частицы в безграничном пространстве в поле потенциальной энергии $U\left(x_{\perp}\right)+U_{\|}\left(x_{\|}\right)$. Легко описать все динамические режимы в таком потенциале. Ускорение центра масс композитной частицы происходит, в точности как у бесструктурной точечной частицы с массой $m_{1}+m_{2}$. Однако внутренняя структура композитной частицы проявляется в характере смены относительных скоростей движения оболочки и внутренней частицы. Так, при захваченном режиме внутренняя частица сталкивается только с одной стороной оболочки, а в пролетном режиме - с обеими сторонами оболочки.

Чтобы завершить обсуждение режимов движения композитной частицы с одной внутренней степенью свободы, обратим внимание на самый сложный случай, когда заряд оболочки сосредоточен на одной из ее граней, например на правой. Разумеется, конфигурационное пространство такой частицы остается прежним. Основное изменение вызвано необходимостью учета кулоновского взаимодействия оболочки и внутренней степени свободы. Это приводит к следующему изменению уравнений движения оболочки и внутренней частицы:

$$
\begin{aligned}
& \sqrt{m_{1}} \ddot{\tilde{x}}_{1}=e_{1} E+e_{1} e_{2}\left|\frac{\tilde{x}_{2}}{\sqrt{m_{2}}}-\frac{\tilde{x}_{1}}{\sqrt{m_{1}}}+\frac{L}{2}\right|^{-2}, \\
& \sqrt{m_{2}} \ddot{\tilde{x}}_{2}=e_{2} E-e_{1} e_{2}\left|\frac{\tilde{x}_{2}}{\sqrt{m_{2}}}-\frac{\tilde{x}_{1}}{\sqrt{m_{1}}}+\frac{L}{2}\right|^{-2} .
\end{aligned}
$$

Из этих уравнений следует, что описание движения композитной частицы конечного размера сводится к описанию движения точечной частицы с упругими отражениями от границ конфигурационного пространства, находящейся под воздействием специального внешнего поля. По этой причине, как и в предыдущем случае, удобно перейти к продольным и поперечным координатам конфигурационного пространства. В этих координатах система уравнений (28) принимает вид

$$
\begin{aligned}
& \ddot{\tilde{x}}_{\|}=\frac{e_{1}+e_{2}}{\sqrt{m_{1}+m_{2}}} E, \\
& \ddot{\tilde{x}}_{\perp}=\frac{1}{\sqrt{m_{1}+m_{2}}}\left\{\frac{e_{2} m_{1}-e_{1} m_{2}}{\sqrt{m_{1} m_{2}}} E-\sqrt{m_{1} m_{2}} \frac{e_{1} e_{2}}{\left|\tilde{x}_{\perp}+\widetilde{L} / 2\right|^{2}}\right\} .
\end{aligned}
$$

Таким образом, мы видим, что продольные и поперечные движения независимы. Это связано с тем, что сила кулоновского взаимодействия зависит только от поперечной координаты. При этом продольные движения сводятся, как и ранее, просто к равноускоренному движению по закону (26) на всех временах эволюции. 


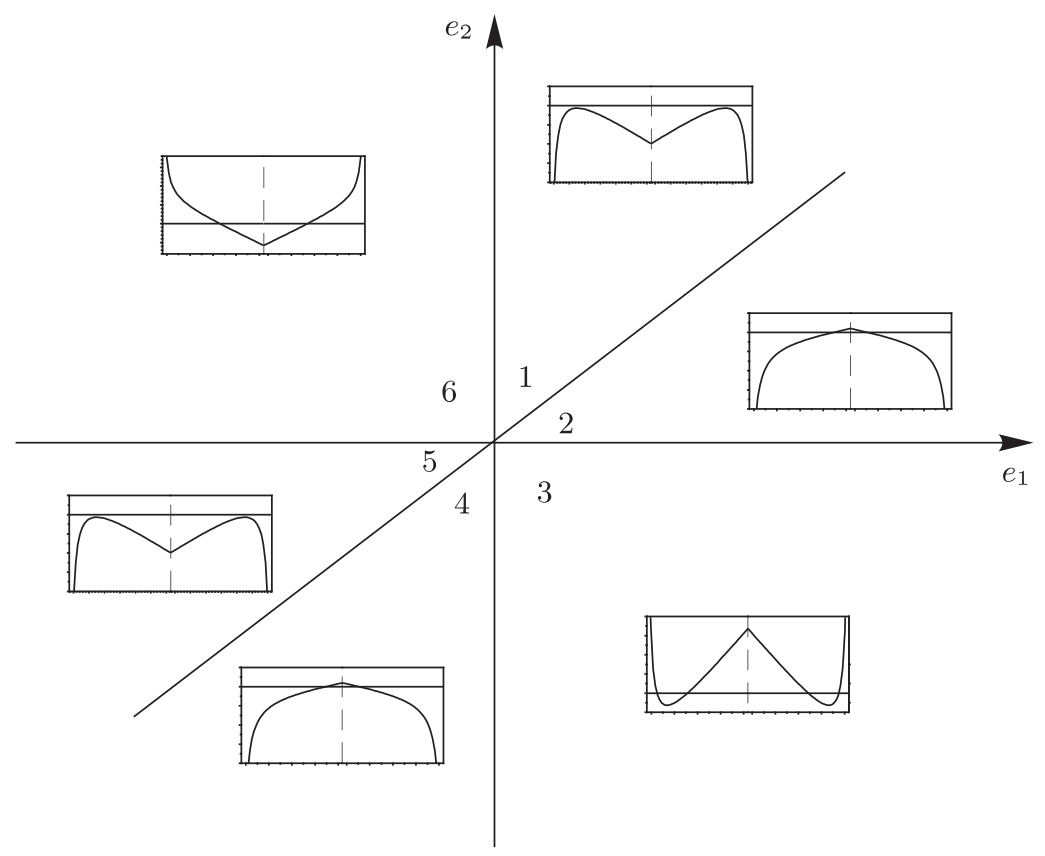

Рис. 8. Бифуркационная диаграмма. Плоскость зарядов $\left(e_{1}, e_{2}\right)$ разделена на секторы, в которых реализуются качественно различные потенциалы внешнего поля. Типичный вид потенциальной энергии на периоде показан в каждом секторе. Наклонная прямая определяется уравнением $e_{2} m_{1}=e_{1} m_{2}$. Данная бифуркационная диаграмма соответствует положительному электрическому полю $E>0$, бифуркационная диаграмма для $E<0$ легко получается из приведенной изменением знаков зарядов на противоположные: $\left(e_{1}, e_{2}\right) \rightarrow\left(-e_{1},-e_{2}\right)$.

Нетривиальные изменения претерпевает эволюция поперечной координаты. Для того чтобы обсудить все возможные режимы движения, удобно снова использовать обобщенный принцип Шварца и перейти от движения в биллиарде к движению в периодическом потенциале в безграничном пространстве. Аналогично изложенному выше введем потенциал внешнего поля, в котором осуществляются поперечные движения. Этот потенциал на интервале $-\widetilde{L} / 2 \leqslant \tilde{x}_{\perp} \leqslant \widetilde{L} / 2$ имеет вид

$$
U\left(\tilde{x}_{\perp}\right)=-\frac{e_{2} m_{1}-e_{1} m_{2}}{\sqrt{m_{1}+m_{2}} \sqrt{m_{1} m_{2}}} E \tilde{x}_{\perp}-\sqrt{m_{1} m_{2}} \frac{e_{1} e_{2}}{\tilde{x}_{\perp}+\widetilde{L} / 2} .
$$

Далее, отражая потенциал внешнего поля при каждом отражении биллиарда относительно соответствующей границы, легко построить искомый периодический потенциал. Таким образом, описание возможных режимов поперечных движений сводится к описанию режимов движения в полученном периодическом потенциале внешнего поля в безграничном пространстве. Ясно, что качественный вид этого потенциала зависит от знаков величин $e_{2} m_{1}-e_{1} m_{2}$ и $e_{1} e_{2}$. 


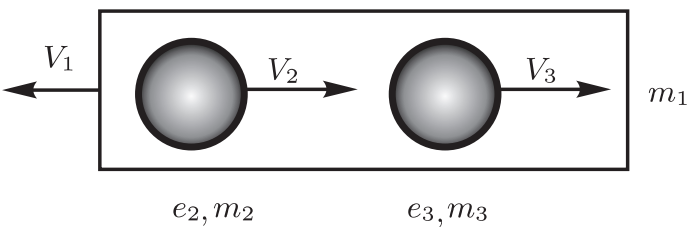

Рис. 9. Композитная частица с двумя внутренними степенями свободы; $V_{2}$, $V_{3}$ - скорости внутренних частиц, $V_{1}$ - скорость оболочки; $m_{2}, m_{3}$ - массы внутренних частиц, $m_{1}$ - масса оболочки. Заряды соответствующих компонент снабжены такими же индексами.

На рис. 8 показана бифуркационная диаграмма возникающих качественно различных потенциалов в зависимости от соотношения зарядов. Опишем все качественно различные режимы эволюции поперечных движений в разных областях бифуркационной диаграммы. Во всех областях, показанных на рис. 8, кроме области 6 , возможны режимы, соответствующие захваченным и пролетным движениям частицы. В области зарядов 6 пролетные режимы отсутствуют. Естественно отметить, что, кроме перечисленных выше, в областях 1 и 5 бифуркационной диаграммы появляется солитоноподобный режим при начальной потенциальной энергии, равной максимуму потенциальной энергии. В этом режиме внутренняя частица сталкивается с одной из сторон оболочки только один раз за все время эволюции. Согласованное ускорение оболочки и внутренней частицы поддерживается кулоновским взаимодействием. Этот режим соответствует движению вдоль сепаратрисы и осуществляется за бесконечное время. Его можно назвать солитоноподобным режимом движения. Для такого движения периодичность в поперечном направлении исчезает. В продольном направлении реализуется равноускоренное движение, а при $e_{1}+e_{2}=0$ - движение с постоянной продольной скоростью (возможно, нулевой).

\section{5. ДВЕ ВНУТРЕННИЕ СТЕПЕНИ СВОБОДЫ}

Перейдем теперь к обсуждению режимов движения композитной частицы с двумя внутреними степенями свободы (см. рис. 9). С ростом числа внутренних степеней свободы значение биллиардного подхода возрастает. Конфигурационное пространство такой частицы определяется неравенствами

$$
\left|x_{2}-x_{1}\right| \leqslant \frac{L}{2}, \quad\left|x_{3}-x_{1}\right| \leqslant \frac{L}{2}
$$

которые означают, что внутренние частицы (их координаты обозначены как $x_{2}, x_{3}$ ) не могут выйти за пределы оболочки (координаты центра оболочки по-прежнему обозначены как $x_{1}$, а ее массу удобно обозначать как $\left.m_{1}\right)$. Еще одно неравенство $x_{2} \leqslant x_{3}$ отражает невозможность прохождения одной частицы сквозь другую.

Переход к биллиардным координатам осуществляется стандартным способом [2]: $\tilde{x}_{i}=\sqrt{m_{i}} x_{i}, i=1,2,3$. В этих координатах конфигурационное пространство представляет собой внутренность трехгранной призмы (см. рис. 10). Действительно, согласно аналитической геометрии [11] трехгранная призма получается в результате 


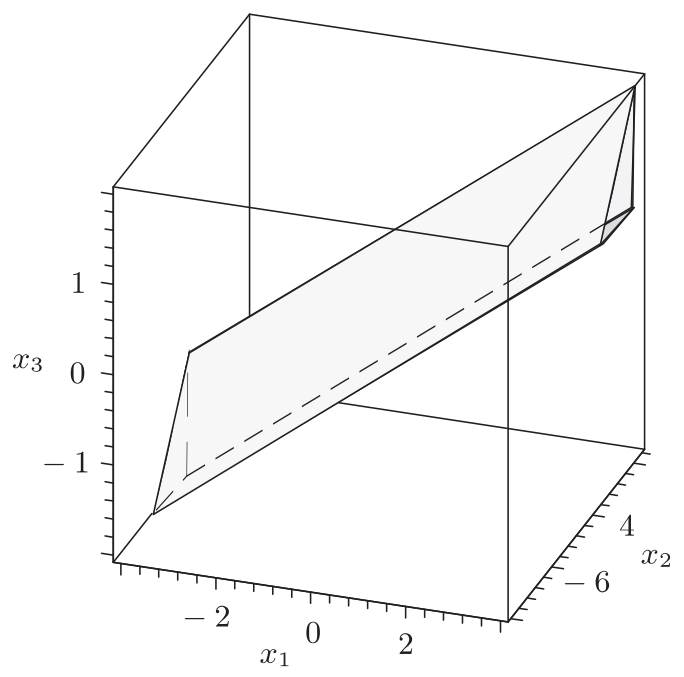

Рис. 10. Конфигурационное пространство - бесконечная треугольная призма.

пересечения трех плоскостей, когда определитель, который составлен из коэффициентов, определяющих уравнения трех плоскостей, обращается в ноль. При этом строки не должны быть пропорциональны. В рассматриваемом случае, записывая уравнения плоскостей в биллиардных координатах, получим уравнение для определителя:

$$
\left|\begin{array}{lll}
A_{1} & B_{1} & C_{1} \\
A_{2} & B_{2} & C_{2} \\
A_{3} & B_{3} & C_{3}
\end{array}\right|=\left|\begin{array}{ccc}
1 / \sqrt{m_{1}} & -1 / \sqrt{m_{2}} & 0 \\
-1 / \sqrt{m_{1}} & 0 & 1 / \sqrt{m_{3}} \\
0 & 1 / \sqrt{m_{2}} & -1 / \sqrt{m_{3}}
\end{array}\right|=0 .
$$

Справедливость этого уравнения очевидна. Здесь и далее мы используем для уравнений $A x_{1}+B x_{2}+C x_{3}+D=0$, определяющих плоскости, стандартные обозначения коэффициентов. Тогда критерий формирования плоскостями трехгранной призмы сводится к условию отличия от нуля по крайней мере одного из определителей [11]

$$
\left|\begin{array}{lll}
A_{1} & B_{1} & D_{1} \\
A_{2} & B_{2} & D_{2} \\
A_{3} & B_{3} & D_{3}
\end{array}\right|, \quad\left|\begin{array}{lll}
A_{1} & C_{1} & D_{1} \\
A_{2} & C_{2} & D_{2} \\
A_{3} & C_{3} & D_{3}
\end{array}\right|, \quad\left|\begin{array}{lll}
B_{1} & C_{1} & D_{1} \\
B_{2} & C_{2} & D_{2} \\
B_{3} & C_{3} & D_{3}
\end{array}\right| .
$$

В выполнении этого условия легко убедиться непосредственной подстановкой значений коэффициентов.

Для дальнейших рассуждений важно установить направление оси призмы. Оно определяется вектором $\vec{\zeta}=\left(\zeta_{1}, \zeta_{2}, \zeta_{3}\right)$, направленным вдоль линии пересечения плоскостей $A_{1} x_{1}+B_{1} x_{2}+C_{1} x_{3}+D_{1}=0$ и $A_{2} x_{1}+B_{2} x_{2}+C_{2} x_{3}+D_{2}=0$ ( см., например, книгу [11]). Компоненты этого вектора задаются соотношениями

$$
\zeta_{1}=\left|\begin{array}{ll}
B_{1} & C_{1} \\
B_{2} & C_{2}
\end{array}\right|, \quad \zeta_{2}=\left|\begin{array}{cc}
C_{1} & A_{1} \\
C_{2} & A_{2}
\end{array}\right|, \quad \zeta_{3}=\left|\begin{array}{ll}
A_{1} & B_{1} \\
A_{2} & B_{2}
\end{array}\right|
$$


Учитывая явный вид элементов, легко вычислить координаты этого вектора:

$$
\vec{\zeta}=-\left(\frac{1}{\sqrt{m_{2} m_{3}}}, \frac{1}{\sqrt{m_{1} m_{3}}}, \frac{1}{\sqrt{m_{1} m_{2}}}\right) .
$$

Направим ось призмы против вектора $\vec{\zeta}$, исключая знак минус, и введем в этом направлении единичный вектор

$$
\vec{\tau}=-\frac{\vec{\zeta}}{|\vec{\zeta}|}=\frac{1}{\sqrt{m_{1}+m_{2}+m_{3}}}\left(\sqrt{m_{1}}, \sqrt{m_{2}}, \sqrt{m_{3}}\right) .
$$

Этот вектор выбран вдоль линии пересечения первых двух плоскостей. Естественно, определение его по пересечению другой пары плоскостей дает то же направление. Таким образом, направление оси треугольной призмы определяется только массами внутренних частиц и оболочки. Выяснив геометрическое устройство границы биллиарда, перейдем к обсуждению движения композитной частицы.

Предварительно следует обсудить распределение зарядов. Будем предполагать, что внутренние точечные частицы имеют заряды $e_{2}$ и $e_{3}$, а заряд оболочки $e_{1}$ равномерно распределен по ней. Это означает, что кулоновское взаимодействие между оболочкой и внутренними частицами отсутствует. Разумеется, величины этих зарядов могут принимать и нулевые значения. Случай $e_{2}=e_{3}=e_{1}=0$, в котором отсутствует влияние электрического поля, был рассмотрен ранее в работе [2], и мы его обсуждать не будем.

Начнем с наиболее простого случая, который соответствует отличным от нуля зарядам оболочки и одной из внутренних частиц. Пусть для определенности $e_{3}=0$, а заряд оболочки $e_{1}$ равномерно распределен по ней. Тогда движение всех компонент такой сложной частицы до столкновения каких-либо ее элементов описывается уравнениями

$$
\begin{aligned}
& \tilde{x}_{1}=\frac{e_{1} E t^{2}}{2 \sqrt{m_{1}}}+\widetilde{V}_{10} t+\tilde{x}_{10} \equiv \frac{e \widetilde{E}_{1} t^{2}}{2 m}+\widetilde{V}_{10} t+\tilde{x}_{10}, \\
& \tilde{x}_{2}=\frac{e_{2} E t^{2}}{2 \sqrt{m_{2}}}+V_{20} t+x_{20} \equiv \frac{e \widetilde{E}_{2} t^{2}}{2 m}+\widetilde{V}_{20} t+\tilde{x}_{20}, \\
& \tilde{x}_{3}=\widetilde{V}_{30} t+\tilde{x}_{30} .
\end{aligned}
$$

Как и ранее, эти уравнения можно интерпретировать как описание в параметрической форме движения одной заряженной точечной частицы в постоянном электрическом поле

$$
\overrightarrow{\widetilde{E}}=\left(\widetilde{E}_{1}, \widetilde{E}_{2}, 0\right), \quad \widetilde{E}_{1}=\frac{e_{1} m}{e \sqrt{m_{1}}} E, \quad \widetilde{E}_{2}=\frac{e_{2} m}{e \sqrt{m_{2}}} E,
$$

где $e$ и $m$ - произвольно выбранные отличные от нуля эталонный заряд и масса. Без ограничения общности их можно считать единичными. Начальные скорости и начальные координаты равны $\widetilde{V}_{i 0}=\sqrt{m_{i}} V_{i 0}$ и $\tilde{x}_{i 0}=\sqrt{m_{i}} x_{i 0}, i=1,2,3$. Начальные координаты $\left(\tilde{x}_{10}, \tilde{x}_{20}, \tilde{x}_{30}\right)$ удобно выбрать принадлежащими границе биллиарда, хотя это и не важно. Важно, что движение эффективной частицы происходит во 
внутренней области биллиарда. Таким образом, движение композитной частицы сводится к движению одной заряженной эффективной частицы в указанном выше биллиарде с зеркальным отражением от границ [2], помещенной в определенное постоянное электрическое поле. Величина и направление этого эффективного поля зависят не только от величины внешнего электрического поля, но и от масс и зарядов внутренних частиц и оболочки.

Перейдем теперь к выяснению динамических свойств частицы, движущейся в таком биллиарде. Прежде всего обратим внимание, что движение эффективной частицы можно разложить на две компоненты: движение вдоль оси биллиарда и поперечное к ней. Ясно, что продольное движение не связано со столкновением частицы со стенкой и является просто равноускоренным движением. Это означает, что продольная скорость $V_{\|}=\vec{V} \cdot \vec{\tau}$ растет пропорционально времени:

$$
V_{\|}=\frac{1}{\sqrt{m_{1}+m_{2}+m_{3}}}\left(\sqrt{m_{1}} \widetilde{V}_{1}+\sqrt{m_{2}} \widetilde{V}_{2}+\sqrt{m_{3}} \widetilde{V}_{3}\right)=\frac{e}{m} \widetilde{E}_{\|} t+\widetilde{V}_{0 \|}
$$

здесь $\widetilde{E}_{\|}=\overrightarrow{\widetilde{E}} \cdot \vec{\tau}$, а $\widetilde{V}_{0 \|}=\overrightarrow{\vec{V}_{0}} \cdot \vec{\tau}-$ начальная продольная скорость. Величина продольного поля зависит от соотношения масс и зарядов компонент композитной частицы:

$$
\widetilde{E}_{\|}=\frac{1}{\sqrt{m_{1}+m_{2}+m_{3}}}\left(\sqrt{m_{1}} \widetilde{E}_{1}+\sqrt{m_{2}} \widetilde{E}_{2}\right) .
$$

Изменение координат в продольном направлении определяется соотношением

$$
\tilde{x}_{\|}=\overrightarrow{\tilde{x}} \cdot \vec{\tau}=\frac{e}{m} \widetilde{E}_{\|} \frac{t^{2}}{2}+\widetilde{V}_{0 \|} t+\tilde{x}_{0 \|}
$$

Вид поперечного электрического поля легко получить:

$$
\begin{aligned}
& \overrightarrow{\widetilde{E}}_{\perp}=\frac{1}{m_{1}+m_{2}+m_{3}} \times \\
& \times\left(\left(m_{2}+m_{3}\right) \widetilde{E}_{1}-\sqrt{m_{1} m_{2}} \widetilde{E}_{2},\left(m_{1}+m_{3}\right) \widetilde{E}_{2}-\sqrt{m_{1} m_{2}} \widetilde{E}_{1},-\sqrt{m_{1} m_{3}} \widetilde{E}_{1}-\sqrt{m_{2} m_{3}} \widetilde{E}_{2}\right) .
\end{aligned}
$$

Обратим внимание, что простейший режим движения в нулевом поперечном поле $\overrightarrow{\widetilde{E}}_{\perp}=0$ невозможен. Легко убедиться, что обращение в ноль поперечного поля приводит к физически нереализуемому условию $m_{1}+m_{2}+m_{3}=0$. Таким образом, режим движения эффективной частицы вдоль оси биллиарда без столкновений с границами биллиарда отсутствует. Для композитной частицы это означает отсутствие режима движения без столкновений между ее компонентами.

Для описания возможных режимов удобно перейти в систему отсчета, движущуюся в продольном направлении со скоростью $V_{\|}$. В этой системе отсчета $V_{\|}=0$ и $\tilde{x}_{\|}=0$. Переход в такую систему отсчета геометрически означает выбор определенного сечения призмы плоскостью $\tilde{x}_{\|}=0$ и проецирование биллиардной траектории на нее. Уравнение, определяющее эту плоскость, есть

$$
\overrightarrow{\tilde{x}} \cdot \vec{\tau}=\frac{m_{1} m_{2} m_{3}}{m_{1}+m_{2}+m_{3}}\left(\frac{\tilde{x}_{1}}{\sqrt{m_{2} m_{3}}}+\frac{\tilde{x}_{2}}{\sqrt{m_{1} m_{3}}}+\frac{\tilde{x}_{3}}{\sqrt{m_{1} m_{2}}}\right)=0 .
$$




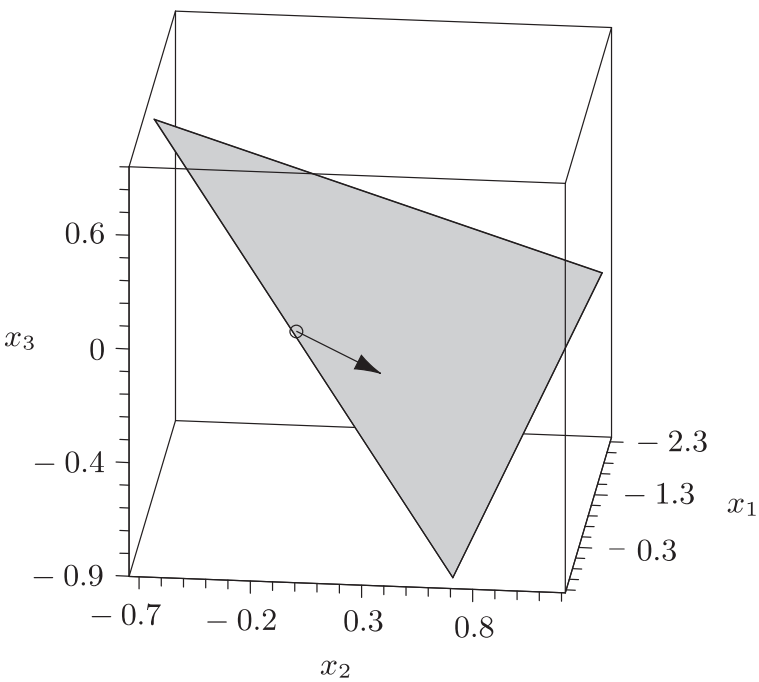

Рис. 11. Сечение призмы плоскостью $\tilde{x}_{\|}=0$ при $m_{1}=1, m_{2}=2, m_{3}=3$ и $L=2$. Область треугольного биллиарда окрашена серым. Направление эффективного электрического поля $\overrightarrow{\widetilde{E}}$ при $e_{1}=1, e_{2}=2$ и $e_{3}=0$ показано стрелочкой.

Таким образом, достаточно следить за проекцией траектории эффективной частицы на это сечение. Сечение треугольной призмы данной плоскостью приводит к треугольному биллиарду (см. рис. 11). Точные координаты вершин треугольника легко вычисляются явно. Они зависят только от масс и размера $L$. Векторы, направленные вдоль сторон этого треугольного биллиарда, определяются линией пересечения соответствующих плоскостей, а именно векторы

$$
\begin{aligned}
& \vec{\xi}_{1}=\left(-\frac{1}{\sqrt{m_{1}}}\left(\frac{1}{m_{2}}+\frac{1}{m_{3}}\right), \frac{1}{m_{3} \sqrt{m_{2}}}, \frac{1}{m_{2} \sqrt{m_{3}}}\right), \\
& \vec{\xi}_{2}=\left(-\frac{1}{m_{2} \sqrt{m_{1}}},-\frac{1}{m_{1} \sqrt{m_{2}}}, \frac{1}{\sqrt{m_{3}}}\left(\frac{1}{m_{2}}+\frac{1}{m_{1}}\right)\right), \\
& \vec{\xi}_{3}=\left(-\frac{1}{m_{3} \sqrt{m_{1}}}, \frac{1}{\sqrt{m_{2}}}\left(\frac{1}{m_{1}}+\frac{1}{m_{3}}\right),-\frac{1}{m_{1} \sqrt{m_{3}}}\right)
\end{aligned}
$$

направлены вдоль сторон, образованных пересечением плоскости (30) с плоскостями

$$
\frac{\tilde{x}_{2}}{\sqrt{m_{2}}}=\frac{\tilde{x}_{3}}{\sqrt{m_{3}}}, \quad \frac{\tilde{x}_{2}}{\sqrt{m_{2}}}-\frac{\tilde{x}_{1}}{\sqrt{m_{1}}}=\frac{L}{2}, \quad \frac{\tilde{x}_{1}}{\sqrt{m_{1}}}-\frac{\tilde{x}_{3}}{\sqrt{m_{3}}}=\frac{L}{2}
$$

соответственно. Используя эти векторы, легко вычислить величины углов треугольного биллиарда (см. рис. 11), которые зависят только от соотношения масс.

Движение проекции траектории в треугольном биллиарде происходит в точности так же, как движение точечной заряженной частицы с зарядом $e$ под воздействием постоянного электрического поля $\vec{E}_{\perp}$ с упругим отражением от границ тре- 


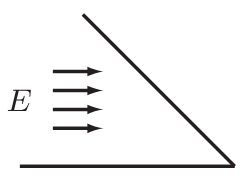

a

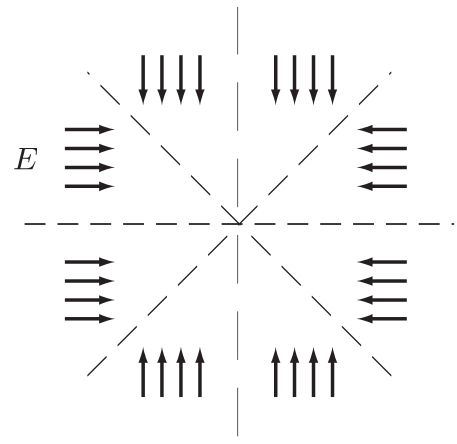

б

Рис. 12. Биллиард в форме угла, равного $\pi / 4$, и направление электрического поля в нем (а); область движения частицы и направления электрического поля после спрямления траектории с использованием обобщенного приема Шварца (б).

угольного биллиарда. Другими словами, для описания всех режимов движения композитной частицы с двумя внутренними степенями свободы достаточно изучить движение заряженной частицы в треугольном биллиарде под действием постоянного электрического поля $\vec{E}_{\perp}$. Напомним, что достижение траекторией одной из границ биллиарда означает столкновение определенной пары объектов. Так, достижение стороны, полученной сечением грани $\tilde{x}_{2} / \sqrt{m_{2}}=\tilde{x}_{3} / \sqrt{m_{3}}$, означает столкновение внутренних частиц; достижение стороны, образованной при сечении грани $\tilde{x}_{1} / \sqrt{m_{1}}-\tilde{x}_{2} / \sqrt{m_{2}}=L / 2,-$ столкновение внутренней частицы с координатой $\tilde{x}_{2}$ с оболочкой. Аналогично, достижение траекторией стороны треугольника, полученной сечением грани $\tilde{x}_{3} / \sqrt{m_{3}}-\tilde{x}_{1} / \sqrt{m_{1}}=L / 2$, означает столкновение внутренней частицы с координатой $\tilde{x}_{3}$ с оболочкой. Ниже мы обсудим качественные черты возможных режимов.

Начнем с обсуждения движения заряженной частицы под воздействием постоянного электрического поля в угле. Это основной элемент движений в треугольном биллиарде (см. рис. 11). Для обычного углового биллиарда справедлива теорема о неизбежном выходе частицы из угла. Эта замечательная теорема и ее обобщения на многомерные углы была доказана Синаем [12].

При наличии электрического поля данная теорема нарушается, если присутствует компонента силы, действующая на частицу в направлении вершины угла. Для того чтобы понять это, рассмотрим простой случай угла, кратного $\pi$. Пусть в угол величиной $\pi / 4$ помещена положительно заряженная частица, имеющая некоторою начальную скорость. Для простоты выберем направление электрического поля параллельно одной из сторон угла (см. рис. 12). Используя обобщение приема Шварца, можно понять, что движение заряженной частицы в угле эквивалентно движению на плоскости в определенном электрическом поле (см. рис. 12). Учитывая, что величина поля постоянна, легко установить вид потенциальной энергии, в которой движется заряженная частица (см. рис. 13). После этого становится очевидным, что движение заряженной частицы в такой потенциальной яме ограничено начальной 


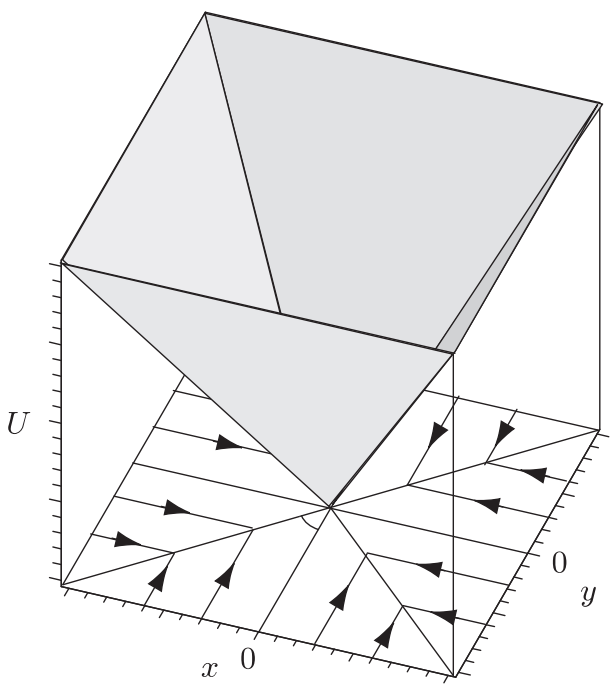

Рис. 13. Вид потенциальной энергии для случая поля, приведенного на рис. 12.

энергией частицы. Все траектории движения частицы финитные, периодические или квазипериодические. Это означает, что частица не выйдет из угла ни при каких начальных условиях.

Интересно отметить, что траектории движения в указанном потенциале находятся аналитически и, как легко понять, складываются из частей парабол. Совершенно аналогично можно доказать, что в случае углов, кратных $\pi$, вылет из угла также не происходит. В этом случае потенциальная энергия усложняется, увеличивается число граней соответствующей пирамиды, а также меняются углы расположения граней. Важно только наличие компоненты силы, действующей на заряженную частицу в направлении вершины угла. Отметим, что обсуждение углов, несоизмеримых с $\pi$, приводит к интересной задаче о поведении частицы в многолистном потенциале.

Для описания хаотических свойств траектории эффективной частицы удобно использовать результаты, полученные при исследовании движения массивной частицы в угловом биллиарде в постоянном гравитационном поле [13]-[15]. В этих работах одна из сторон углового биллиарда ориентирована вдоль постоянного гравитационного поля. Так, в работе [13] доказано, что в зависимости от величины угла реализуется хаотическое поведение с положительным показателем Ляпунова. При этом значение показателя Ляпунова и доля площади хаотических режимов на плоскости сечения Пуанкаре осциллируют с изменением величины угла. Для углов $0<\theta<\pi / 4$ наблюдается типичное поведение типа Колмогорова-Арнольда-Мозера, соответствующее островкам устойчивости в хаотическом море. При $\theta=\pi / 4$ динамика точно интегрируема. И, наконец, при $\pi / 4<\theta<\pi / 2$ возникает сильный хаос [13]-[15]. Разумеется, все эти свойства сохраняются и для постоянного электрического поля, ориентированного специальным образом (вдоль одной из сторон угла).

В общем случае электрическое поле ориентировано произвольно по направлению к вершине угла. Естественно, следует ожидать, что хаотические режимы сохранятся 
и в этом случае. Интересно отметить, что для анализа поведения траекторий в угловых биллиардах в постоянном гравитационном поле также полезно использовать обобщенный прием Щварца, предложенный в настоящей работе. Это позволило бы перейти к движению частицы в специальном внешнем поле, но в безграничном пространстве.

Перечисленные выше свойства приводят к некоторому набору возможных режимов в треугольном биллиарде, при которых траектория заперта в соответствующем угле и никогда не достигает стороны треугольника, противоположной вершине этого угла. Такие режимы реализуются при полях, больших некоторого критического значения. Критическое значение поля определяется из условия равенства кинетической энергии частицы минимальному значению потенциальной энергии электрического поля, которая достигается над противолежащей стороной треугольника. При заданном направлении поля это критическое значение легко вычисляется.

Таким образом, появляются режимы движения композитной частицы в постоянном электрическом поле, при которых в процессе движения внутренние частицы сталкиваются хаотически с оболочкой без столкновений между собой. Подобное поведение возникает, когда эффективное электрическое поле $\overrightarrow{E_{\perp}}$ направлено к углу, образованному сторонами треугольника, которые соответствуют столкновению внутренней частицы с оболочкой.

Если эффективное электрическое поле $\vec{E}_{\perp}$ направлено к вершине, образованной сторонами, одна из которых определяет столкновение одной внутренней частицы с оболочкой, а вторая - столкновение внутренних частиц, то в этом случае реализуется другой режим. В этом режиме внутренняя частица хаотически сталкивается с оболочкой или со второй внутренней частицей. При этом вторая внутренняя частица не сталкивается с оболочкой. Во всех этих режимах должно наблюдаться хаотическое движение оболочки. Как мы видели, величина углов определяется соотношением масс внутренних частиц и оболочки. Таким образом, соотношение масс и определяет "степень" хаотичности. Разумеется, в этих условиях присутствуют также всевозможные периодические режимы, анализ которых для углового биллиарда в гравитационном поле можно найти в работе [15]. В соответствии с известными общими свойствами хаотических режимов гамильтоновых систем в хаотическом море расположено счетное количество периодических орбит.

С уменьшением величины электрического поля биллиардная траектория в треугольном биллиарде достигает также противолежащей стороны, поэтому становятся возможными режимы движения, при которых хаотически сталкиваются все элементы композитной частицы.

Разумеется, в случае силы, действующей на частицу от вершины угла, выход частицы из угла неизбежен, как и при отсутствии поля. В этом случае яма превращается в барьер, “скатывание” с которого ускоряет выход из угла. Можно сказать, что вход в угол ограничен барьером. Кроме этого, интересно заметить, что при увеличении электрического поля и, следовательно, при увеличении угла наклона эффективного потенциала возникает своеобразный вторичный биллиард, который характеризует траектории в угле. Так, например, в рассмотренном выше примере с углом $\pi / 4$ задача в нулевом приближении сводится к движению частицы в квадратном биллиарде. Все типы траекторий такого биллиарда достаточно хорошо изучены [16], его топологическая энтропия равна нулю [17], и хаотические траектории отсутствуют. Учет наклона потенциальной ямы в следующем приближении ведет 
к мягким биллиардам [18]-[20]. Такие биллиарды отличаются характером отражения: в точке отражения возникает дуга, а не излом траектории, соответствующий закону “угол падения равен углу отражения". Таким образом, для описания движения заряженной частицы в угле и постоянном поле в определенном приближении можно использовать обычные биллиарды в многоугольниках без внешних полей.

Интересно отметить, что хаотичность угловых биллиардов в постоянном поле означает, что движение частицы в пирамидальных потенциалах довольно простого вида (введенных выше) будет хаотичным. В результате мы имеем простые примеры классических систем с нарушенной гладкостью и, возможно, с многолистной потенциальной энергией, приводящие к развитому хаотическому движению.

Возвращаясь к треугольному биллиарду в постоянном поле, можно продвинуться достаточно далеко, если использовать результаты об обычном биллиарде с треугольной границей. В частности, рассмотрим такие треугольные биллиарды, углы которых рационально соизмеримы с $\pi$. Другими словами, пусть углы при вершинах треугольника равны $\varphi_{i}=\pi m_{i} / n_{i}$, где $n_{i}$ и $m_{i}$ - целые числа, $i=1,2,3$. Наименьший общий знаменатель этих углов обозначим через $N$. Тогда род поверхности $S$, которую можно получить, замостив ее $2 N$ копиями треугольника с использованием принципа Шварца, определяется соотношением [21]

$$
g(S)=1+\frac{N}{2} \sum_{i=1}^{3} \frac{m_{i}-1}{n_{i}}
$$

Теперь учтем наличие постоянного электрического поля. Обобщенный принцип Шварца приводит к появлению на этой поверхности дополнительного специального электрического поля, элементарной ячейкой которого служит треугольник. В результате возникает движение заряженной частицы по поверхности рода $g$ в заданном потенциале.

$\mathrm{B}$ частности, для треугольника с углами $\varphi_{1}=\pi / 2, \varphi_{2}=\varphi_{3}=\pi / 4$ род равен 1 и поверхность $S$ соответствует тору $T^{2}$. Развертка этого тора с указанием направлений постоянного электрического поля приведена на рис. 14. Движение заряженной частицы будет происходить на плоскости в двухпериодическом потенциале. В данном случае потенциал в элементарной квадратной ячейке соответствует потенциалу, приведенному на рис. 13, и реализуются два типа движений, отвечающие пролетным и захваченным режимам.

Обратим внимание, что значение максимумов потенциальной энергии в приведенном примере зависит от направления электрического поля. Так, при направлении поля, ортогональном к границе развертки, максимум потенциала, который достигается на границе развертки, одинаков во всех точках. При нарушении ортогональности максимумы достигаются по-прежнему на границе развертки, но теперь величина локального максимума зависит от выбора точки. Это явление типично для любого набора углов треугольника. Такая зависимость максимумов от направлений приводит к специфической чувствительности поведения решения к начальным условиям, физический механизм которой связан с разрезанием потока начальных условий на одном энергетическом уровне на части, одна из которых отражается от потенциального барьера, а вторая преодолевает этот барьер. После такого разрезания близкие траектории расходятся на значительное расстояние. Поэтому в таких системах может возникать хаотическое поведение по механизму разрезания потоков, подобно 


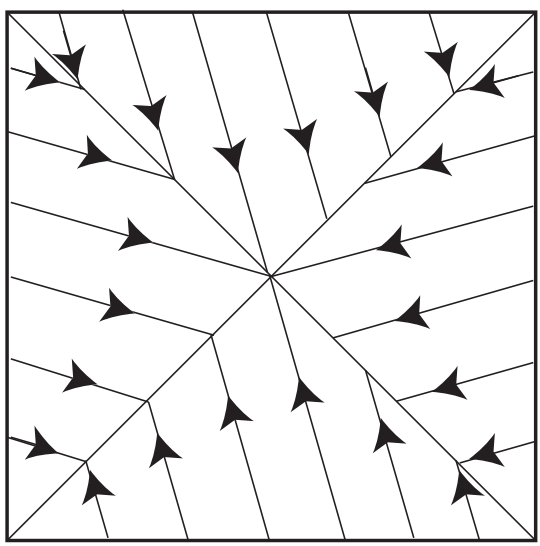

Рис. 14. Развертка тора с указанием направлений постоянного электрического поля на нем. Тонкими линиями показано покрытие квадрата исходным треугольником. Тор возникает после попарного склеивания противоположных сторон.

рассмотренному в работе [22]. Таким образом, даже в случае значений угла, при которых отсутствует хаос, для траекторий в угле в треугольном биллиарде могут появляться хаотические режимы.

Биллиардный подход применим и к движению заряженной композитной частицы с любым числом внутренних степеней свободы в постоянном электрическом поле. В настоящей работе мы ограничились для наглядности только малым числом внутренних степеней свободы. С ростом этого числа усложняется как форма многомерного биллиарда, так и множество возможных режимов движения.

\section{6. ЗАКЛЮЧЕНИЕ}

Обсудим динамические свойства рассмотренных композитных частиц в постоянном электрическом поле. Общим свойством является равноускоренное движение центра масс при отличном от нуля суммарном заряде композитной частицы. При равенстве суммарного заряда нулю центр масс сохраняет свою скорость. Однако, говоря о динамике композитных частиц, следует иметь в виду, что наблюдаемой компонентой является оболочка композитной частицы. Движение внутренних степеней свободы, как правило, не контролируется. Поэтому основные выводы о динамике композитных частиц сформулируем в терминах движения оболочки.

В соответствии с полученными результатами для композитной частицы с одной степенью свободы в поведении оболочки могут наблюдаться следующие режимы. Прежде всего, ускорение оболочки может происходить по двум сценариям. По одному из них ускорение оболочки происходит периодически на определенный "квант" скорости. При втором сценарии присутствуют два "кванта" скорости, один увеличивает скорость, второй уменьшает ее так, что после одного периода скорость оболочки возрастает. Период этих изменений определяется характеристиками оболочки и внутренней частицы, как показано выше. 
Такая простая динамика наблюдается, если заряжена только внутренняя частица. Если заряжена и оболочка, то характер ее движений зависит от характера распределения заряда по ней. Если заряд однородно распределен по оболочке, то характер ее движения сводится к равноускоренному движению с периодической дискретной компонентой изменения скорости, как и в вышеописанном случае. При заряде, локализованном в некоторой точке оболочки, дополнительное влияние оказывает кулоновское взаимодействие, и тогда при полученных выше условиях возникает еще один режим. В этом режиме исчезает периодическая компонента изменения скорости оболочки и характер ускорения оболочки отличается от равноускоренного.

При увеличении числа внутренних степеней свободы основное изменение в поведении оболочки даже при одной заряженной внутренней частице сводится к появлению хаотических режимов, приводящих к ее ускорению. Также становятся возможными и более сложные периодические компоненты ускорения с большим разнообразием величин, на которые происходит изменение скорости оболочки на одном периоде.

Отметим, что наличие периодических компонент может быть интересно с точки зрения создания излучающих наноустройств, особенно в случае нулевого суммарного заряда, когда излучающее устройство может покоиться и излучать под воздействием постоянного электрического поля. В случае общего положения спектр излучения такого излучателя качественно формируется из излучения равноускоренного заряда, периодического движения заряда и компоненты, напоминающей тормозное излучение при столкновении оболочки и внутренней частицы. Детальное изучение излучения таких частиц требует отдельного рассмотрения и выходит за рамки настоящей работы.

\section{Список литературы}

[1] А. И. Гусев, Наноматериаль, наноструктуры, нанотехнологии, Физматлит, М., 2005.

[2] В. В. Яновский, А. В. Тур, Ю. Н. Масловский, ЖЭТФ, 133:1 (2008), 220-236.

[3] B. W. Smith, M. Monthioux, D. E. Luzzi, Nature, 396:6709 (1998), 323-324.

[4] M. Monthioux, Carbon, 40:10 (2002), 1809-1823.

[5] K. Suenaga, T. Okazaki, K. Hirahara, S. Bandow, H. Kato, A. Taninaka, H. Shinohara, S. Iijima, Appl. Phys. A, 76:4 (2003), 445-447.

[6] Г. Шилл, Катенаны, ротаксаны и узлы, Мир, М., 1973.

[7] I. T. Harrison, S. Harrison, J. Amer. Chem. Soc., 89:22 (1967), 5723-5724.

[8] J. W. Choi, A. H. Flood, D. W. Steuerman, S. Nygaard, A. B. Braunschweig, N. N. P. Moonen, B. W. Laursen, Y. Luo, E. DeIonno, A. J. Peters, J. O. Jeppesen, K. Xu, J. F. Stoddart, J. R. Heath, Chem. Eur. J., 12:1 (2006), 261-279.

[9] J. E. Green, J. W. Choi, A. Boukai, Y. Bunimovich, E. Johnston-Halperin, E. DeIonno, Y. Luo, B. A. Sheriff, K. Xu, Y.S. Shin, H. Tseng, J. F. Stoddart, J. R. Heath, Nature, 445:7126 (2007), 414-417.

[10] Г. А. Гальперин, Н. И. Чернов, Биллиарды и хаос, Знание, М., 1991.

[11] Б. Н. Делоне, Д. А. Райков, Аналитическая геометрия, т. 2, Гостехиздат, М.-Л., 1949.

[12] Я. Г. Синай, УМН, 33:1(199) (1978), 229-230.

[13] P. H. Richter, H.-J. Scholz, A. Wittek, Nonlinearity, 3:1 (1990), 45-67.

[14] N. D. Whelan, D. A. Goodings, J. K. Cannizzo, Phys. Rev. A, 42:2 (1990), 742-754.

[15] T. Szeredi, D. A. Goodings, Phys. Rev. E, 48:5 (1993), 3518-3528.

[16] Г. А. Гальперин, А. Н. Земляков, Математические билъярды, Наука, М., 1990. 
[17] E. Gutkin, H. Haydn, Bull. Amer. Math. Soc. (N.S.), 32:1 (1995), 50-56.

[18] P. R. Baldwin, Physica D, 29:3 (1988), 321-342.

[19] D. Turaev, V. Rom-Kedar, J. Statist. Phys., 112:3-4 (2003), 765-813.

[20] H. A. Oliveira, C. Manchein, M. W. Beims, Phys. Rev. E, 78:4 (2008), 046208, 9 pp., arXiv: 0903.0170.

[21] E. Gutkin, Physica D, 19:3 (1986), 311-333.

[22] S. V. Slipushenko, A. V. Tur, V. V. Yanovskij, Изв. вузов. Прикл. нелин. динам., 18:4 (2010), 91-110.

Поступила в редакцию 28.09.2012, после доработки 28.12.2012 